\title{
On the uniform controllability of the Burgers equation
}

\author{
O. Glass* \& S. Guerrero*
}

July 25, 2006

\begin{abstract}
In this paper, we deal with the viscous Burgers equation with a small dissipation coefficient $\nu$. We prove the (global) exact controllability property to nonzero constant states, that is to say, the possibility of finding boundary values such that the solution of the associated Burgers equation is driven to a constant state. The main objective of this paper is to do so with control functions whose norms in an appropriate space are bounded independently of $\nu$, which belongs to a suitably small interval. This result is obtained for a sufficiently large time.
\end{abstract}

\section{Introduction}

\subsection{Statement of the result and background}

We are interested in the controllability of the Burgers equation in a bounded interval:

$$
u_{t}+u u_{x}-\nu u_{x x}=0 \text { in }(0, T) \times(0,1)
$$

where $T$ is a positive real number. We complete this equation with the following: we give an initial condition

$$
u_{\mid t=0}=u_{0} \text { in }(0,1)
$$

and controlled boundary values:

$$
u_{\mid x=0}=v_{1} \text { in }(0, T), \quad u_{\mid x=1}=v_{2} \text { in }(0, T) .
$$

Here, $v_{1}$ and $v_{2}$ stand for control functions, which translates in the possibility of acting over the system through both endpoints of the boundary $x=0$ and $x=1$. Let $\bar{u}$ be a solution of (1) with Dirichlet boundary conditions (also called a trajectory). The exact controllability to trajectories holds if we can find controls $v_{1}$ and $v_{2}$ such that the associated solution coincides with $\bar{u}$ at time $t=T$. In this paper, we are interested in proving an exact controllability result uniformly with respect to the viscosity coefficient $\nu$ in a sufficiently small range, when $\bar{u}$ is constant.

Let us be more specific on the problem under view. We consider the system constituted by (1), (2) and (3). Let us fix the initial condition $u_{0}$ in some Banach space $X$ and a constant $M \neq 0$; then, our goal is to find two controls $v_{1}$ and $v_{2}$ such that the associated solution $u$ satisfies

$$
\left.u\right|_{t=T}=M \text { in }(0,1) .
$$

Moreover, as we said above we are interested in finding controls whose norms in some Banach space $Y$ are uniformly bounded with respect to $\nu$, whenever $\nu$ is sufficiently small and $T$ is sufficiently large. The main result of this paper is given in the following theorem, where we prove the previous results for $X=L^{\infty}(0,1)$ and $Y=L^{\infty}(0, T)$ :

*Université Pierre et Marie Curie-Paris6, UMR 7598 Laboratoire Jacques-Louis Lions, Paris, F-75005 France. E-mails: glass@ann.jussieu.fr, guerrero@ann.jussieu.fr. 
Theorem 1. There is a constant $\alpha_{0} \geq 1$ such that for any $M \in \mathbb{R} \backslash\{0\}$ there exists $\nu_{0}>0$ such that for any $u_{0} \in L^{\infty}(0,1)$, any time $T>\alpha_{0} /|M|$ and any $\nu \in\left(0, \nu_{0}\right)$ there exist controls $v_{1}^{\nu}$ and $v_{2}^{\nu}$ satisfying the following properties:

- $\left\|v_{1}^{\nu}\right\|_{\infty}$ and $\left\|v_{2}^{\nu}\right\|_{\infty}$ are uniformly bounded for $\nu \in\left(0, \nu_{0}\right)$, that is to say, there exists a constant $C\left(\alpha_{0}\right)>0$ such that

$$
\left\|v_{1}^{\nu}\right\|_{\infty}+\left\|v_{2}^{\nu}\right\|_{\infty} \leq C\left(\left\|u_{0}\right\|_{\infty}+|M|\right)
$$

- The solution $u$ of (1)-(2)-(3) associated to $v_{1}=v_{1}^{\nu}$ and $v_{2}=v_{2}^{\nu}$ satisfies (4).

The controllability of Burgers equation for fixed $\nu$ has been studied by several authors. In particular, two kinds of controllability properties have been considered:

- On the one hand, the local exact controllability to trajectories, which stands for the concept of exact controllability with the additional assumption that the initial state $u_{0}$ is close to the initial state of the targeted trajectory $\bar{u}_{\mid t=0}$; this has been established for the Burgers equation in [8]. It is also proved in [8] that the global exact controllability to stationary trajectories does not hold as long as one controls in a sub-interval $(a, b)$ of $(0,1)$, which is equivalent to control at one endpoint. In the more recent work [9], the authors prove that the global exact controllability for (1) does not hold even if the control is acting on both sides of the domain.

- On the other hand, in [4] the author establishes a global result between 0 and constant states: precisely, the author proves that for $u_{0}=0$ and for any $T>0$ one can drive the solution of (1) to any constant $M$ satisfying that $|M|$ is sufficiently large with respect to $T$.

Here, as we are interested in the properties of uniform controllability as $\nu \rightarrow 0^{+}$, it seems natural to regard the inviscid framework $(\nu=0)$. In this case and in the context of entropy solutions, the controllability of the equation

$$
u_{t}+\left(u^{2} / 2\right)_{x}=0
$$

was studied in [12], where some conditions are given on the final state in order to ensure this property. More general convex scalar conservation laws

$$
u_{t}+(f(u))_{x}=0
$$

were considered in [1], for which the controllability problem is posed in the half line with null initial condition. The set of attainable states is completely described.

We recall that for conservation laws such as (5), solutions generally develop singularities in finite time, regardless of the regularity of the initial condition. This leads to considering distributional solutions, but in this setting, uniqueness is lost. From both physical and mathematical standpoints, it is then natural to consider solutions of (6) that fulfill entropy conditions in order to extract the physically relevant solution. These are the following: for any regular couple $(\eta, q)$ defined on $\mathbb{R}$ and such that $\eta^{\prime} f^{\prime}=q^{\prime}$ and $\eta$ is convex, the following stands in the sense of measures:

$$
\eta(u)_{t}+q(u)_{x} \leq 0
$$

We underline that entropy solutions are the ones which can be obtained by vanishing viscosity: one can summarize the situation by saying that the viscosity has disappeared from the equation, and is only effective for the selection of admissible discontinuities. Concerning the Cauchy problem, equation (1) was first approached by Hopf [11], in which an explicit formula is given, and the limit as $\nu \rightarrow 0^{+}$is considered. The convergence of vanishing viscosity approximations to the entropy solutions of a general scalar conservation law was studied in the celebrated work of Kruzkov [13]. For a general reference to conservation laws, we refer to [6].

It is therefore very natural, when considering control problems for conservation laws, to consider the cost of the viscosity, that is, to determine if known controllability properties for the hyperbolic equation are still valid for the model with small viscosity, and how the size of the control evolves as the viscosity approaches 0 . Note that some problems such as the global approximate controllability of the Navier-Stokes equation with Navier slip boundary conditions [3], are obtained through controllability results for the inviscid equation (in this case, the Euler equation). 


\subsection{Some remarks}

Let us make some remarks on the above theorem, and state a corollary which concerns the system with $\nu=1$.

Remark 1. In general, entropy solutions of (5) cannot reach a state $M$ (starting for instance from $\left.u_{0}=0\right)$, in a time less than $1 /|M|$. In particular, the state 0 cannot be reached unless one has $u_{0}=0$. This is easily seen by considering generalized backward characteristics (see [1]). Hence the time of control $O(1 / M)$ is not surprising. Note that even in the case of a linear transport equation, the uniform controllability results [5, 10] consider a time of control of the form $C /|M|$, $C>1$.

Remark 2. Following the proof of Theorem 1, one can check that Theorem 1 holds for the choices $\alpha_{0}=7$ (or in fact, 6.3) and

$$
\nu_{0}=\nu_{1} \min \{1,|M| /|\log (|M|)|\},
$$

where $\nu_{1}$ is a small enough constant independent of $M$ but depending on $\alpha_{0}$.

Now we can present the following result as a consequence of Theorem 1.

Corollary 1. Consider for $w^{0} \in L^{\infty}(0,1)$ and $T_{0}>0$ the following control problem:

$$
\begin{cases}w_{t}+w w_{x}-w_{x x}=0 & \text { in }\left(0, T_{0}\right) \times(0,1), \\ w_{\mid x=0}=\tilde{v}_{1}(t), \quad w_{\mid x=1}=\tilde{v}_{2}(t) & \text { in }\left(0, T_{0}\right) \\ w_{\mid t=0}=w_{0} & \text { in }(0,1) .\end{cases}
$$

Assume that $\left|M_{0}\right|$ is large enough in order that

$$
\left|M_{0}\right| \nu_{1} \geq\left|\log \left(\left|M_{0}\right| \nu_{1}\right)\right|
$$

where $\nu_{1}$ is defined in (7); then, for every $T_{0}>\alpha_{0} /\left|M_{0}\right|$ (where $\alpha_{0}$ is chosen as in Remark 2), there exist controls $\tilde{v}_{1}(t)$ and $\tilde{v}_{2}(t)$ in $L^{\infty}(0, T)$ such that the solution of (8) satisfies

$$
w_{\mid t=T_{0}}=M_{0} .
$$

Note in particular that Corollary 1 implies that the result of $[4]$ is valid for any $u_{0} \in L^{\infty}(0,1)$. Let us also underline that the time that we use to control the system depends only on the final state, and is independent from the initial one.

Proof. This is a simple scaling argument. Indeed, let us set

$$
\left\{\begin{array}{l}
u(t, x)=\nu_{1} w\left(\nu_{1} t, x\right), v_{1}(t)=\nu_{1} \tilde{v}_{1}\left(\nu_{1} t\right), v_{2}(t)=\nu_{1} \tilde{v}_{2}\left(\nu_{1} t\right) \text { and } u_{0}(x)=\nu_{1} w_{0}(x) . \\
t \in(0, T), x \in(0,1)
\end{array}\right.
$$

where we have denoted $T=T_{0} / \nu_{1}$. Then, we have

$$
\begin{cases}u_{t}+u u_{x}-\nu_{1} u_{x x}=0 & \text { in }(0, T) \times(0,1), \\ u_{\mid x=0}=v_{1}(t), w_{\mid x=1}=v_{2}(t) & \text { in }(0, T) \\ u_{\mid t=0}=w_{0} & \text { in }(0,1) .\end{cases}
$$

Let us set $M:=\nu_{1} M_{0}$. From Theorem 1 and since $T>\alpha_{0} /|M|$ (thanks to the choice of $T_{0}$ ) and $\nu_{1} \leq \nu_{1} \min \{1,|M| /|\log | M||\}$ (thanks to the choice of $M_{0}$ ), we know the existence of $v_{1}$ and $v_{2}$ such that the solution of (10) satisfies $\left.u\right|_{t=T}=M$ in $(0,1)$. Going back to $w$, this shows the existence of two controls $\tilde{v}_{1}$ and $\tilde{v}_{2}$ such that the associated solution of (8) satisfies $\left.w\right|_{t=T_{0}}=M_{0}$ in $(0,1)$, as we wanted to prove. 


\subsection{Structure of the paper}

One of the main ingredients of the proof is the use of the return method by J.-M. Coron, which consists in finding a particular trajectory of the system which moves far away from the initial state to get back to the final state afterward. In the present situation we steer the system to a large constant state $N$, and then we get back to the constant state $M$.

Consequently, the proof of Theorem 1 is divided in two parts, which we summarize in the following propositions.

Proposition 1. There are some constants $\alpha_{1} \geq 1$ and $\nu_{1}>0$ such that: for any $u_{0} \in L^{\infty}(0,1)$, for any $N \in \mathbb{R}$ with $|N|$ large enough and any $\nu \in\left(0, \nu_{1}\right)$ there are controls $w_{1}^{\nu}$ and $w_{2}^{\nu}$ in $L^{\infty}\left(0, T_{1}\right)$, where $T_{1}=\alpha_{1} /|N|$, satisfying the following properties:

- $\left\|w_{1}^{\nu}\right\|_{\infty}$ and $\left\|w_{2}^{\nu}\right\|_{\infty}$ are uniformly bounded for $\nu \in\left[0, \nu_{1}\right]$.

- The associated solution $u$ satisfies $u_{\mid t=T_{1}}=N$ in $(0,1)$.

Proposition 2. The conclusion of Theorem 1 is true when $M>0$ and $u_{0}$ is a positive constant large enough with respect to $M$.

The planning of the paper is the following: Proposition 1 is established in Section 2. Section 3 is devoted to prove Proposition 2 and finally, we prove some technical results we need for the previous propositions in Section 4.

\section{Proof of Proposition 1}

Due to the invariance of the solutions of (1) by the transformation $u(t, x) \leftrightarrow-u(t, 1-x)$, we can assume that $N>0$. Now the proof of Proposition 1 is divided in two parts. First, we prove that we can reach a state close to $N$ in a time $O(1 / N)$ (which is a kind of global approximate controllability, but where the target is a constant that depends on the initial one) and then we prove that we can steer the latter state exactly to $N$ in a time $O(1 / N)$ (local exact controllability).

\subsection{Reaching $N$ approximately}

In the following proposition we prove that, starting from a $L^{\infty}$ initial condition, we can construct a solution of (1)-(2) which is close in $W^{1, \infty}$ norm to some large constant.

Proposition 3. Given $u_{0} \in L^{\infty}(0,1)$, one can find $N>0$ large enough such that for any $\nu>0$, one can find controls $v_{1}$ and $v_{2}$ such that the solution of (1)-(2)-(3) satisfies:

$$
\|u(t, \cdot)-N\|_{L^{\infty}(0,1)} \leq\left(\left\|u_{0}\right\|_{\infty}+N / 2\right) \exp \left\{-\frac{3 N^{2}}{16 \nu}\left(t-\frac{8}{N}\right)\right\},
$$

for any $t>0$ and

$$
\left\|u_{x}(t, \cdot)\right\|_{L^{\infty}(0,1)} \leq C \frac{N^{2}}{\nu} \exp \left\{-\frac{3 N^{2}}{16 \nu}\left(t-\frac{8}{N}\right)\right\}
$$

for any $t>8 / N$ and some $C>0$. Moreover, the controls satisfy independently from $\nu$ :

$$
\max \left(\left\|v_{1}\right\|_{L^{\infty}(0, T)},\left\|v_{2}\right\|_{L^{\infty}(0, T)}\right) \leq N .
$$

Remark 3. All the above constants (such as 8 or 16) are not optimal (see the proof below) but are sufficient for our purpose (because $N$ is arbitrarily large).

\section{Proof of Proposition 3.}

The proof of this proposition relies on the comparison principle and on traveling waves for equation (1). Let us state precisely the comparison principle for the reader's convenience. 
Lemma 1 (Comparison principle). Consider $\bar{u}_{1}$ and $\bar{u}_{2}$ in $L^{\infty}(\mathbb{R})$, and the corresponding solutions $u_{1}$ and $u_{2}$ of the Burgers equation on the whole real line with initial conditions $\bar{u}_{1}$ and $\bar{u}_{2}$, respectively. Then, if

$$
\bar{u}_{1} \leq \bar{u}_{2} \text { in } \mathbb{R}
$$

we have

$$
u_{1}(t, x) \leq u_{2}(t, x) \text { in } \mathbb{R}^{+} \times \mathbb{R}
$$

A simple way to prove Lemma 1 (although it could be proven in a far more general setting) is to use Hopf's formula for solutions of the viscous Burgers equation [11]:

$$
u_{i}(t, x)=\frac{\int_{-\infty}^{\infty} \frac{x-y}{t} \exp \left\{-\frac{1}{2 \nu}\left(\frac{(x-y)^{2}}{2 t}+\int_{0}^{y} \bar{u}_{i}(\eta) d \eta\right)\right\} d y}{\int_{-\infty}^{\infty} \exp \left\{-\frac{1}{2 \nu}\left(\frac{(x-y)^{2}}{2 t}+\int_{0}^{y} \bar{u}_{i}(\eta) d \eta\right)\right\} d y} \quad i=1,2 .
$$

We consider the function

$$
\rho_{i}(x, y):=\exp \left\{-\frac{1}{2 \nu}\left(\frac{(x-y)^{2}}{2 t}+\int_{0}^{y} \bar{u}_{i}(\eta) d \eta\right)\right\}
$$

and $d \mu_{i}$ the probability measure (depending on $x$ ) given by

$$
d \mu_{i}:=\frac{\rho_{i}(x, y)}{\int_{-\infty}^{\infty} \rho_{i}(x, \cdot)} d y
$$

Now we have

$$
\begin{aligned}
u_{2}(t, x) & =\int_{-\infty}^{+\infty} \frac{x-y}{t} d \mu_{2} \\
& =\int_{-\infty}^{+\infty} \frac{x-y}{t} \exp \left(-\frac{1}{2 \nu} \int_{0}^{y}\left(\bar{u}_{2}(\eta)-\bar{u}_{1}(\eta)\right) d \eta\right) d \mu_{1} \times \frac{\int_{-\infty}^{+\infty} \rho_{1}(x, y) d y}{\int_{-\infty}^{+\infty} \rho_{2}(x, y) d y} \\
& \geq \int_{-\infty}^{+\infty} \frac{x-y}{t} d \mu_{1} \times \int_{-\infty}^{+\infty} \exp \left(-\frac{1}{2 \nu} \int_{0}^{y}\left(\bar{u}_{2}(\eta)-\bar{u}_{1}(\eta)\right) d \eta\right) d \mu_{1} \times \frac{\int_{-\infty}^{+\infty} \rho_{1}(x, y) d y}{\int_{-\infty}^{+\infty} \rho_{2}(x, y) d y} \\
& =\int_{-\infty}^{+\infty} \frac{x-y}{t} d \mu_{1}=u_{1}(t, x)
\end{aligned}
$$

The inequality used above is the FKG inequality: for $\mu$ a probability measure on $\mathbb{R}$ and two non-decreasing functions $f$ and $g$, one has:

$$
\int_{\mathbb{R}} f g d \mu \geq \int_{\mathbb{R}} f d \mu \times \int_{\mathbb{R}} g d \mu .
$$

(This follows easily by considering $\iint(f(x)-f(y))(g(x)-g(y)) d \mu(x) d \mu(y)$.)

Back to the proof of Proposition 3. We introduce travelling wave profiles for equation (1). These are solutions of the viscous Burgers equations on the whole real line, of the form:

$$
u(t, x)=U(x-c t) \quad t \in \mathbb{R}^{+}, x \in \mathbb{R},
$$

with $c$ a fixed real number which will be chosen later on. Furthermore, $u$ satisfies the following asymptotic properties:

$$
u(t, x) \longrightarrow U^{-} \text {as } x \rightarrow-\infty, u(t, x) \longrightarrow U^{+} \text {as } x \rightarrow+\infty, \quad u_{x}(t, x) \longrightarrow 0 \text { as } x \rightarrow \pm \infty,
$$


where $U^{-}$and $U^{+}$are constant states. Straightforward computations show that these travelling waves are given by the following:

$$
\begin{gathered}
U^{-} \geq U^{+}, \\
c=\frac{U^{-}+U^{+}}{2}, \\
U(y)=\frac{U^{-}+U^{+}}{2}-\frac{U^{-}-U^{+}}{2} \tanh \left(\frac{U^{-}-U^{+}}{2 \nu}\left(y-y_{0}\right)\right),
\end{gathered}
$$

where of course $y_{0}$ is arbitrary (we will refer to $y_{0}$ as the center of the wave).

Now let us go back to the proof of Proposition 3. Given $u_{0}$, we choose $N>2\left\|u_{0}\right\|_{\infty}$; later we will also take $N>L\left\|u_{0}\right\|_{\infty}$ for some $L \geq 2$ and in Section 3 we will additionally require $N>M$ ). We introduce:

- $u$ as the solution of the Burgers equation on $\mathbb{R}$ with initial value

$$
u(0, x)=\left\{\begin{array}{l}
N \text { for } x<0, \\
u_{0}(x) \text { for } 0 \leq x \leq 1, \\
0 \text { for } x>1
\end{array}\right.
$$

- $\check{u}$ as the travelling wave solution of the Burgers equation with $U^{-}=N$ and $U^{+}=-2\left\|u_{0}\right\|_{\infty}$, initially centered at $y_{0}$.

The goal is to prove that the restriction of $u$ to $[0,1]$ is a suitable solution for Proposition 3 (of course, $v_{1}$ and $v_{2}$ are defined as the traces of $u$ at $x=0$ and $x=1$, respectively). Now if $y_{0}$ is such that

$$
\check{u}(0, \cdot) \leq u_{0}(\cdot) \text { in }(0,1),
$$

it follows from the comparison principle that

$$
\check{u}(t, x) \leq u(t, x) \leq N \text { in } \mathbb{R}^{+} \times \mathbb{R} .
$$

Let us do a choice of $y_{0}$ so that (21) is satisfied. Indeed, if we take

$$
y_{0}=-\frac{2 \nu}{N+2\left\|u_{0}\right\|_{\infty}} \operatorname{arctanh}\left(\frac{N}{N+2\left\|u_{0}\right\|_{\infty}}\right),
$$

one can easily check that

$$
\check{u}(0, x) \leq-\left\|u_{0}\right\|_{\infty} \quad \forall x \in[0,1],
$$

just taking into account that the maximum value of the function

$$
x \in[0,1] \longmapsto-\tanh \left(\frac{U^{-}-U^{+}}{2 \nu}\left(x-y_{0}\right)\right)
$$

is reached at $x=0$.

Clearly, for $N$ large enough, one has

$$
y_{0} \geq-1
$$

and

$$
N-2\left\|u_{0}\right\|_{\infty} \geq \frac{N}{2} .
$$

Let us now prove that estimate (11) holds. We first recall the expression of $\check{u}$ :

$$
\left\{\begin{array}{l}
\check{u}(t, x)=\frac{N}{2}-\left\|u_{0}\right\|_{\infty}-\frac{N+2\left\|u_{0}\right\|_{\infty}}{2} \tanh \left(\frac{N+2\left\|u_{0}\right\|_{\infty}}{2 \nu}\left(x-\frac{N-2\left\|u_{0}\right\|_{\infty}}{2} t-y_{0}\right)\right) \\
t \in \mathbb{R}^{+}, x \in \mathbb{R} .
\end{array}\right.
$$


From (23) and (24), we get

$$
\begin{aligned}
& \tanh \left(\frac{N+2\left\|u_{0}\right\|_{\infty}}{2 \nu}\left(x-\frac{N-2\left\|u_{0}\right\|_{\infty}}{2} t-y_{0}\right)\right) \leq \tanh \left(\frac{N+2\left\|u_{0}\right\|_{\infty}}{2 \nu}\left(-\frac{N}{4} t+2\right)\right) \\
& \leq \tanh \left(\frac{-3 N^{2}}{16 \nu}\left(t-\frac{8}{N}\right)\right) \quad t \in \mathbb{R}^{+}, x \in[0,1] .
\end{aligned}
$$

On the other hand, from the definition of the function tanh, we readily deduce that

$$
\tanh \left(\frac{-3 N^{2}}{16 \nu}\left(t-\frac{8}{N}\right)\right) \leq-1+\exp \left\{\frac{-3 N^{2}}{16 \nu}\left(t-\frac{8}{N}\right)\right\} .
$$

Going back to (25), we obtain

$$
\check{u}(t, x)-N \geq-\left(\frac{N}{2}+\left\|u_{0}\right\|_{\infty}\right) \exp \left\{\frac{-3 N^{2}}{16 \nu}\left(t-\frac{8}{N}\right)\right\} .
$$

Since $\check{u}(t, x)-N \leq 0$ already holds (see (22)), we deduce (11).

In order to prove (12) we begin by giving an explicit representation of the spatial derivative of $u$ in the following lemma:

Lemma 2. Let $u$ be a solution of

$$
\begin{cases}u_{t}-\nu u_{x x}+u u_{x}=0 & (t, x) \in \mathbb{R}_{+} \times \mathbb{R} \\ u_{\mid t=0}=u_{0} & x \in \mathbb{R} .\end{cases}
$$

Then one has

$$
\partial_{x} u(t, x)=\frac{1}{2 \nu} \frac{\int_{-\infty}^{+\infty} \frac{y-x}{t}\left(u_{0}(y)-u(t, x)\right) \exp \left\{-\frac{1}{2 \nu}\left[\frac{(x-y)^{2}}{2 t}+\int_{0}^{y} u_{0}(\eta) d \eta\right]\right\} d y}{\int_{-\infty}^{+\infty} \exp \left\{-\frac{1}{2 \nu}\left[\frac{(x-y)^{2}}{2 t}+\int_{0}^{y} u_{0}(\eta) d \eta\right]\right\} d y} .
$$

Proof. Let us define

$$
f(t, x):=\int_{-\infty}^{+\infty} \frac{x-y}{t} \exp \left\{-\frac{1}{2 \nu}\left[\frac{(x-y)^{2}}{2 t}+\int_{0}^{y} u_{0}(\eta) d \eta\right]\right\} d y
$$

and

$$
g(t, x):=\int_{-\infty}^{+\infty} \exp \left\{-\frac{1}{2 \nu}\left[\frac{(x-y)^{2}}{2 t}+\int_{0}^{y} u_{0}(\eta) d \eta\right]\right\} d y .
$$

From Hopf's formula (see (16)), we find

$$
u(t, x)=\frac{f(t, x)}{g(t, x)} \quad(t, x) \in \mathbb{R}_{+} \times \mathbb{R} .
$$

We notice that $\partial_{x} g=-\frac{1}{2 \nu} f$ and

$$
\partial_{x} f(t, x)=\frac{g}{t}-\frac{1}{2 \nu t} \int_{-\infty}^{+\infty} \frac{(y-x)^{2}}{t} \exp \left\{-\frac{1}{2 \nu}\left[\frac{(x-y)^{2}}{2 t}+\int_{0}^{y} u_{0}(\eta) d \eta\right]\right\} d y
$$

so that

$$
\partial_{x} u(t, x)=\frac{1}{t}-\frac{1}{2 \nu}\left[\frac{\int_{-\infty}^{+\infty} \frac{(y-x)^{2}}{t^{2}} \exp \left\{-\frac{1}{2 \nu}\left[\frac{(x-y)^{2}}{2 t}+\int_{0}^{y} u_{0}(\eta) d \eta\right]\right\} d y}{g}-\frac{f^{2}}{g^{2}}\right]
$$


Now let us consider the second term in the above right hand side:

$$
\begin{aligned}
I:= & \int_{-\infty}^{+\infty} \frac{(y-x)^{2}}{t^{2}} \exp \left\{-\frac{1}{2 \nu}\left[\frac{(x-y)^{2}}{2 t}+\int_{0}^{y} u_{0}(\eta) d \eta\right]\right\} d y \\
= & \int_{-\infty}^{+\infty} \frac{y-x}{t}\left(\frac{y-x}{t}+u_{0}(y)\right) \exp \left\{-\frac{1}{2 \nu}\left[\frac{(y-x)^{2}}{2 t}+\int_{0}^{y} u_{0}(\eta) d \eta\right]\right\} d y \\
& -\int_{-\infty}^{+\infty} \frac{y-x}{t} u_{0}(y) \exp \left\{-\frac{1}{2 \nu}\left[\frac{(y-x)^{2}}{2 t}+\int_{0}^{y} u_{0}(\eta) d \eta\right]\right\} d y \\
= & \frac{2 \nu g}{t}-\int_{-\infty}^{+\infty} \frac{y-x}{t} u_{0}(y) \exp \left\{-\frac{1}{2 \nu}\left[\frac{(y-x)^{2}}{2 t}+\int_{0}^{y} u_{0}(\eta) d \eta\right]\right\} d y,
\end{aligned}
$$

In the last identity we have used the fact that

$$
\begin{aligned}
& -2 \nu \frac{d}{d y} \exp \left\{-\frac{1}{2 \nu}\left[\frac{(y-x)^{2}}{2 t}+\int_{0}^{y} u_{0}(\eta) d \eta\right]\right\} \\
& =\left(\frac{y-x}{t}+u_{0}(y)\right) \exp \left\{-\frac{1}{2 \nu}\left[\frac{(y-x)^{2}}{2 t}+\int_{0}^{y} u_{0}(\eta) d \eta\right]\right\}
\end{aligned}
$$

and we have integrated by parts. Injecting $I$ in (27) yields

$$
\begin{aligned}
\partial_{x} u(t, x)=-\frac{1}{2 \nu g}\left[-\int_{-\infty}^{+\infty} \frac{y-x}{t} u_{0}(y) \exp \left\{-\frac{1}{2 \nu}\left[\frac{(y-x)^{2}}{2 t}+\int_{0}^{y} u_{0}(\eta) d \eta\right]\right.\right. & d y \\
& -u(t, x) f(t, x)]
\end{aligned}
$$

which yields (26).

Back to the proof of (12). We consider $x \in[0,1]$ and use (26) to estimate $\partial_{x} u(t, x)$ :

$$
\begin{aligned}
\partial_{x} u(t, x) & =\frac{1}{2 \nu} \frac{\int_{-\infty}^{+\infty} \frac{y-x}{t}\left(u_{0}(y)-N\right) \exp \left\{-\frac{1}{2 \nu}\left[\frac{(x-y)^{2}}{2 t}+\int_{0}^{y} u_{0}(\eta) d \eta\right]\right\} d y}{\int_{-\infty}^{+\infty} \exp \left\{-\frac{1}{2 \nu}\left[\frac{(x-y)^{2}}{2 t}+\int_{0}^{y} u_{0}(\eta) d \eta\right]\right\} d y} \\
& +\frac{1}{2 \nu} \frac{\int_{-\infty}^{+\infty} \frac{y-x}{t}(N-u(t, x)) \exp \left\{-\frac{1}{2 \nu}\left[\frac{(x-y)^{2}}{2 t}+\int_{0}^{y} u_{0}(\eta) d \eta\right]\right\} d y}{\int_{-\infty}^{+\infty} \exp \left\{-\frac{1}{2 \nu}\left[\frac{(x-y)^{2}}{2 t}+\int_{0}^{y} u_{0}(\eta) d \eta\right]\right\} d y}
\end{aligned}
$$

Let us denote $A$ and $B$ respectively the first and second term of the above right hand side. Clearly,

$$
B=\frac{1}{2 \nu}(N-u(t, x)) u(t, x),
$$

and this term is easily estimated using the $L^{\infty}$ estimate on $N-u$. Concerning $A$, we first notice that, due to the initial condition of $u$ (see (20)), one has

$$
A=\frac{1}{2 \nu} \frac{\int_{0}^{+\infty} \frac{y-x}{t}\left(u_{0}(y)-N\right) \exp \left\{-\frac{1}{2 \nu}\left[\frac{(x-y)^{2}}{2 t}+\int_{0}^{y} u_{0}(\eta) d \eta\right]\right\} d y}{\int_{-\infty}^{+\infty} \exp \left\{-\frac{1}{2 \nu}\left[\frac{(x-y)^{2}}{2 t}+\int_{0}^{y} u_{0}(\eta) d \eta\right]\right\} d y} .
$$

Note that simple computations yield

$$
\int_{a}^{b} \exp \left\{-\frac{1}{2 \nu}\left[\frac{(x-y)^{2}}{t}+\alpha y\right]\right\} d y=2 \sqrt{\nu t} \exp \left\{\frac{\alpha(-2 x+\alpha t)}{4 \nu}\right\} \int_{\xi_{-}}^{\xi_{+}} e^{-t^{2}} d t
$$


with

$$
\xi_{-}:=\frac{a-x+\alpha t}{2 \sqrt{\nu t}} \text { and } \xi_{+}:=\frac{b-x+\alpha t}{2 \sqrt{\nu t}}
$$

and

$$
\begin{aligned}
\int_{a}^{b} y \exp \left\{-\frac{1}{2 \nu}\right. & {\left.\left[\frac{(x-y)^{2}}{t}+\alpha y\right]\right\} d y=2 \sqrt{\nu t}(x-\alpha t) \exp \left\{\frac{\alpha(-2 x+\alpha t)}{4 \nu}\right\} \int_{\xi_{-}}^{\xi_{+}} e^{-t^{2}} d t } \\
& -2 \nu t\left\{\exp \left[-\frac{b^{2}-2 b x+2 b \alpha t+x^{2}}{4 \nu t}\right]-\exp \left[-\frac{a^{2}-2 a x+2 a \alpha t+x^{2}}{4 \nu t}\right]\right\} .
\end{aligned}
$$

Also, we note that for $y>0$, one has

$$
\int_{y}^{+\infty} e^{-s^{2}} d s \leq \frac{e^{-y^{2}}}{2 y}
$$

We estimate from below the denominator by

$$
\begin{aligned}
\int_{-\infty}^{+\infty} \exp \left\{-\frac{1}{2 \nu}\left[\frac{(x-y)^{2}}{2 t}+\int_{0}^{y} u_{0}(\eta) d \eta\right]\right\} d y & \geq \int_{-\infty}^{0} \exp \left\{-\frac{1}{2 \nu}\left[\frac{(x-y)^{2}}{2 t}+\int_{0}^{y} u_{0}(\eta) d \eta\right]\right\} d y \\
& =\int_{-\infty}^{0} \exp \left\{-\frac{1}{2 \nu}\left[\frac{(x-y)^{2}}{2 t}+N y\right]\right\} d y \\
& \geq \sqrt{\pi \nu t} \exp \left\{\frac{N}{2 \nu}\left(\frac{N t}{2}-x\right)\right\} .
\end{aligned}
$$

The numerator is bounded by

$$
\begin{aligned}
& \left|\int_{0}^{+\infty} \frac{y-x}{t}\left(u_{0}(y)-N\right) \exp \left\{-\frac{1}{2 \nu}\left[\frac{(x-y)^{2}}{2 t}+\int_{0}^{y} u_{0}(\eta) d \eta\right]\right\} d y\right| \\
\leq & \int_{0}^{+\infty} \frac{y+1}{t}\left(\left\|u_{0}\right\|_{\infty}+N\right) \exp \left\{-\frac{1}{2 \nu}\left[\frac{(x-y)^{2}}{2 t}-\left\|u_{0}\right\|_{\infty} y\right]\right\} d y \\
\leq & 12 \nu N \exp \left\{\frac{-x^{2}}{4 \nu t}\right\},
\end{aligned}
$$

where we used $N>\left\|u_{0}\right\|_{\infty}$ and $N t-1>1$ for the times under view.

Then (12) follows by taking $N>>\left\|u_{0}\right\|_{\infty}$. Note that the estimate on the term $A$ is better than the estimate on the term $B$, which comes directly from the $L^{\infty}$ estimate.

Finally, estimate (13) comes directly from the maximum principle.

Remark 4. It is easy to check the following: suppose that we take $N$ large enough (as we may) in order that

$$
\begin{gathered}
y_{0} \geq-\delta, \\
N-2\left\|u_{0}\right\|_{\infty} \geq(1-\delta) N,
\end{gathered}
$$

for some small parameter $\delta>0$, instead of (23)-(24). Then following the above computations we obtain estimates of the form

$$
\|u(t, \cdot)-N\|_{W^{1, \infty}(0,1)} \leq \frac{C N^{2}}{\nu} \exp \left\{-\frac{K}{\nu}\left(t-\frac{2(1+\delta)}{N(1-\delta)}\right)\right\} .
$$

Hence the time of approximate controllability can be made close to $2 / N$ for $N$ large enough (as $\left.\nu \rightarrow 0^{+}\right)$. This is not surprising, since for the hyperbolic equation, the solution of the Riemann problem (5) with initial condition

$$
\left.u\right|_{t=0}=\left\{\begin{array}{l}
N \text { on } \mathbb{R}^{-}, \\
M \text { on } \mathbb{R}^{+},
\end{array}\right.
$$

with $N, M \in \mathbb{R}, N>M$ is given by a simple shock with speed $(N+M) / 2$. 


\subsection{Reaching $N$ exactly}

Observe that from the previous paragraph and after a time $t=\alpha_{1} / N$ has gone by (with for instance $\alpha_{1}=8$ or even $\alpha_{1}>2$, taking Remark 4 into account), we can assume that our new initial condition (which we also denote $u_{0}$ ) satisfies

$$
\left\|u_{0}-N\right\|_{W^{1, \infty}(0,1)} \leq e^{-C N / \nu}
$$

for some $C>0$, provided that $\nu \in\left(0, \nu_{0}\right)$.

Remark 5. As said above, condition (31) trivially follows from Proposition 3, since $\nu$ is small enough and $N$ is large enough (in particular, $\nu_{0}=\nu_{1}$ in remark 2 above), but we have given it here because condition (31) also holds for $M$ instead of $N$ as long as $\nu_{0}$ is taken like

$$
\nu_{0}=\nu_{1}\{1, M /|\log (|M|)|\}
$$

for some $\nu_{1}>0$ small enough (see remark 2 for more details).

In this paragraph, we prove that we have a local exact (uniform in $\nu$ ) controllability result for a time $T=O(1 / N)$. Precisely, we establish:

Proposition 4. Assume that $u_{0} \in W^{1, \infty}(0,1)$ and there exists $K_{0}>0$ such that

$$
\left\|u_{0}-N\right\|_{W^{1, \infty}(0,1)} \leq e^{-K_{0} N / \nu} .
$$

Then, one can find controls $v_{1}$ and $v_{2}$ such that the solution of (1)-(2)-(3) satisfies, for $T=\frac{4.3}{N}$,

$$
\left.u\right|_{t=T}=N \quad \text { in }(0,1) .
$$

Moreover, the controls satisfy the following estimate, which is independent of $\nu \in\left(0, \nu_{0}\right)$ :

$$
\max \left(\left\|v_{1}\right\|_{W^{1, \infty}(0, T)},\left\|v_{2}\right\|_{W^{1, \infty}(0, T)}\right) \leq 2 N .
$$

Proof: First, we set $y(t, x)=u(t, x)-N$ so that $y$ fulfills

$$
\begin{cases}y_{t}+y y_{x}-\nu y_{x x}+N y_{x}=0 & \text { in }(0, T) \times(0,1) \\ \left.y\right|_{t=0}=y^{0}:=u^{0}-N & \text { in }(0,1) .\end{cases}
$$

Now, our objective is to find boundary controls $\left.y\right|_{x=0}(t)=v_{1}(t)-N$ and $\left.y\right|_{x=1}(t)=v_{2}(t)-N$ such that

$$
\left.y\right|_{t=T}=0 \text { in }(0,1)
$$

and

$$
\left\|v_{1}(t)-N\right\|_{W^{1, \infty}(0, T)}+\left\|v_{2}(t)-N\right\|_{W^{1, \infty}(0, T)} \leq N .
$$

We will prove this by means of a fixed point argument posed in a suitable Hilbert space $Z$.

\subsubsection{Uniform null controllability of the linearized Burgers equation}

In this paragraph, we consider the following linearized Burgers equation:

$$
\begin{cases}y_{t}-\nu y_{x x}+((N+z(t, x) / 2) y)_{x}=0 & \text { in }(0, T) \times(0,1), \\ \left.y\right|_{x=0}=\widetilde{v}_{1},\left.\quad y\right|_{x=1}=\widetilde{v}_{2} & \text { in }(0, T), \\ \left.y\right|_{t=0}=y^{0} & \text { in }(0,1) .\end{cases}
$$

For this system, we prove the null controllability with controls bounded independently of $\nu$. Precisely, we have: 
Lemma 3. Let $z$ be in $L^{1}\left(0, T ; W^{1, \infty}(0,1)\right) \cap L^{\infty}((0, T) \times(0,1))$ with

$$
\|z\|_{\infty} \leq \exp \left(-\frac{K_{0} N}{5 \nu}\right)
$$

and let us introduce the quantity

$$
D(T, N, z):=\frac{3 e^{-2\left\|z_{x}\right\|_{L_{t}^{1}\left(L_{x}^{\infty}\right)}}}{4}\left(\frac{2 T\left(N-\|z\|_{\infty} / 2\right)}{3}-1\right)^{2}-2.61 .
$$

Assume that the initial condition $y^{0}=u^{0}-N$ satisfies (32) and that the final time $T$ satisfies

$$
\left(N-\|z\|_{\infty} / 2\right) 2 T / 3>1 .
$$

Then, there exists a positive constant $K_{2}>0$ such that for any $\nu \in\left(0, \nu_{0}\right)$, there exist two controls $\widetilde{v}_{1}, \widetilde{v}_{2} \in W^{1, \infty}(0, T)$ such that the associated solution to (38) satisfies

$$
\left.y\right|_{t=T}=0 \quad \text { in }(0,1)
$$

and

$$
\left\|\widetilde{v}_{1}\right\|_{W^{1, \infty}(0, T)}+\left\|\widetilde{v}_{2}\right\|_{W^{1, \infty}(0, T)} \leq e^{-K_{0} N /(3 \nu)}\left(e^{-D\left(T^{*}, N, z\right) /\left(\nu T^{*}\right)}+1\right),
$$

where $T^{*}:=\min \left\{T, 3 /\left(N-\|z\|_{\infty} / 2\right)\right\}$.

Proof of Lemma 3: First, choosing the controls $\widetilde{v}_{1}$ and $\widetilde{v}_{2}$ to be zero close to $t=0$ and on account of the regularizing effect of the heat equation, one can always suppose that our initial condition $y^{0}$ belongs to $W^{2, \infty}(0,1)$ and, thanks to $(32)$, we can also assume that

$$
\left\|y^{0}\right\|_{W^{2, \infty}(0,1)} \leq e^{-K_{0} N /(2 \nu)} .
$$

Now, we introduce a function $\widetilde{y}^{0} \in W^{2, \infty}(-1,2)$ such that $\widetilde{y}^{0}=y^{0}$ in $(0,1)$ and

$$
\left\|\widetilde{y}^{0}\right\|_{W^{2, \infty}(-1,2)} \leq C\left\|y^{0}\right\|_{W^{2, \infty}(0,1)}
$$

for some $C>0$. Let us first suppose that we can find two controls $\widetilde{v}_{3}, \widetilde{v}_{4} \in L^{2}(0, T)$ satisfying

$$
\begin{aligned}
\left\|\widetilde{v}_{3}\right\|_{L^{2}\left(0, T^{*}\right)}+\left\|\widetilde{v}_{4}\right\|_{L^{2}\left(0, T^{*}\right)} & \leq C e^{-D\left(T^{*}, N, z\right) /\left(\nu T^{*}\right)}\left\|\widetilde{y}^{0}\right\|_{L^{2}(-1,2)} \\
& \leq C e^{-D\left(T^{*}, N, z\right) /\left(\nu T^{*}\right)} e^{-K_{0} N /(2 \nu)}
\end{aligned}
$$

for some $C>0$, such that the solution $\widetilde{y} \in L^{2}((0, T) \times(-1,2))$ of

$$
\begin{cases}\widetilde{y}_{t}-\nu \widetilde{y}_{x x}+((N+z(t, x) / 2) \widetilde{y})_{x}=0 & \text { in }\left(0, T^{*}\right) \times(-1,2), \\ \left.\widetilde{y}\right|_{x=-1}=\widetilde{v}_{3},\left.\quad \widetilde{y}\right|_{x=2}=\widetilde{v}_{4} & \text { in }\left(0, T^{*}\right), \\ \left.\widetilde{y}\right|_{t=0}=\widetilde{y}^{0} & \text { in }(-1,2),\end{cases}
$$

satisfies

$$
\left.\widetilde{y}\right|_{t=T^{*}}=0 \quad \text { in }(-1,2) .
$$

Then, the function $y:=\left.\widetilde{y}\right|_{[0,1]} \mathbb{1}_{\left(0, T^{*}\right)}$ fulfills system (38) with

$$
\widetilde{v}_{1}(t)=\left.\widetilde{y}\right|_{x=0}(t) \mathbb{1}_{\left(0, T^{*}\right)} \quad \text { and } \quad \widetilde{v}_{2}(t)=\left.\widetilde{y}\right|_{x=1}(t) \mathbb{1}_{\left(0, T^{*}\right)}
$$

and satisfies

$$
\left.y\right|_{t=T}=0 \quad \text { in }(0,1)
$$

(indeed, $y \equiv 0$ in $\left(T^{*}, T\right)$ ). 
In order to prove estimate (42), it suffices to use classical localization arguments together with regularity estimates for the solution of (46). Precisely, for any $\delta>0$, we can prove that there exists a positive constant $C>0$ such that

$$
\|\widetilde{y}\|_{W^{1, \infty}\left(0, T ; H^{1}(-1+\delta, 2-\delta)\right)} \leq(C / \nu)\left(\left\|\widetilde{y}^{0}\right\|_{W^{2, \infty}(-1,2)}+\left\|\widetilde{v}_{3}\right\|_{L^{2}\left(0, T^{*}\right)}+\left\|\widetilde{v}_{4}\right\|_{L^{2}\left(0, T^{*}\right)}\right) .
$$

In particular, this implies that $\left.\widetilde{y}\right|_{x=0}$ and $\left.\widetilde{y}\right|_{x=1}$ belong to $W^{1, \infty}\left(0, T^{*}\right)$ and thanks to (45), (32) and $\nu \in\left(0, \nu_{0}\right)$, they satisfy estimate $(42)$.

Consequently, our task will be now to find $\widetilde{v}_{3}$ and $\widetilde{v}_{4}$ satisfying the above properties. We use the classical approach, consisting of obtaining a suitable observability inequality for the adjoint system of (46). For simplicity, we will suppose that we are working in the space interval $(0,1)$ instead of $(-1,2)$ (so, in particular, we will refer to system (38) instead of (46)). Thus, let us introduce the adjoint problem associated to (38):

$$
\begin{cases}-\varphi_{t}-\nu \varphi_{x x}-(N+z(t, x) / 2) \varphi_{x}=0 & \text { in }\left(0, T^{*}\right) \times(0,1) \\ \left.\varphi\right|_{x=0}=0,\left.\quad \varphi\right|_{x=1}=0 & \text { in }\left(0, T^{*}\right), \\ \left.\varphi\right|_{t=T^{*}}=\varphi^{0} & \text { in }(0,1)\end{cases}
$$

where, $\varphi^{0} \in H_{0}^{1}(0,1)$ is the initial condition.

We prove the following observability inequality for the solutions of (48):

$$
\left\|\left.\varphi\right|_{t=0}\right\|_{L^{2}(0,1)}^{2} \leq K\left(T^{*}, \nu\right) \int_{0}^{T^{*}}\left(\left.\left|\varphi_{x}\right|_{x=0}\right|^{2}+\left.\left|\varphi_{x}\right|_{x=1}\right|^{2}\right) d t
$$

for some positive constant $K\left(T^{*}, \nu\right)$. Then, it is not difficult to prove that the null controllability of system (38) holds with controls $\widetilde{v}_{1}$ and $\widetilde{v}_{2}$ whose $L^{2}$ norms are bounded by $K\left(T^{*}, \nu\right) / \nu$. We omit the proof of this fact for the sake of simplicity.

In order to prove estimate (49), we will follow the same ideas in [5]. That is to say, we will combine a suitable Carleman inequality with a dissipation result for system (48).

Dissipation Result: Let $t_{0} \in\left(0, T^{*}\right)$. Then, following the steps of the proof in [7], one can prove:

$$
\left\{\begin{array}{c}
\left\|\left.\varphi\right|_{t=t_{0}}\right\|_{L^{2}(0,1)}^{2} \\
\quad \leq \exp \left\{-\frac{\left(\left(N-\|z\|_{\infty} / 2\right) t^{*}-1\right)^{2}}{2 \nu t^{*}} e^{-2\left\|z_{x}\right\|_{L_{t}^{1}\left(L_{x}^{\infty}\right)}}\right\}\left\|\left.\varphi\right|_{t=t_{0}+t^{*}}\right\|_{L^{2}(0,1)}^{2},
\end{array}\right.
$$

for any $t^{*} \in\left(0, T^{*}-t_{0}\right)$ such that

$$
\left(N-\|z\|_{\infty} / 2\right) t^{*}>1 .
$$

Carleman inequality: Let $0<\gamma<1 / 3$ (one can take for example $\gamma:=1 / 6$ ). We will prove the following inequality:

$$
\int_{0}^{1} \int_{2 T^{*} / 3}^{(2+3 \gamma) T^{*} / 3}|\varphi|^{2} d t d x \leqslant C e^{(2.61) /\left(\nu T^{*}\right)}\left(\frac{\nu^{2} T^{*}}{N} \int_{0}^{T^{*}}\left|\varphi_{x}(t, 0)\right|^{2} d t+\frac{1}{N} \int_{0}^{1}|\varphi(0, x)|^{2} d x\right)
$$

The proofs of these results are postponed to the fourth and last section of the paper.

Now, we are in position to establish our central observability inequality (49). We apply the dissipativity result (50) to $\varphi$ (which is a solution of (48)) for $t^{*} \in\left(2 T^{*} / 3,(2+3 \gamma) T^{*} / 3\right)$. This is possible by (41). We obtain

$$
\left\{\begin{aligned}
\int_{0}^{1} \int_{2 T^{*} / 3}^{(2+3 \gamma) T^{*} / 3} & |\varphi|^{2} d t d x \\
& \geq \exp \left\{3 e^{-2\left\|z_{x}\right\|_{L_{t}^{1}\left(L_{x}^{\infty}\right)}}\left(\left(2 T^{*} / 3\right)\left(N-\|z\|_{\infty} / 2\right)-1\right)^{2} /\left(4 \nu T^{*}\right)\right\} \int_{0}^{1}|\varphi(0, x)|^{2} d x .
\end{aligned}\right.
$$


Here, we have used the fact that

$$
t^{*} \longmapsto \frac{\left(\left(N-\|z\|_{\infty} / 2\right) t^{*}-1\right)^{2}}{4 \nu t^{*}}
$$

is an increasing function as long as (51) is satisfied.

Finally, we obtain the observability inequality (49) with

$$
K\left(T^{*}, \nu\right)=C \exp \left(-\frac{D\left(T^{*}, N, z\right)}{\nu T^{*}}\right)
$$

for some $C=C\left(T^{*}, N\right)$; recall that $D\left(T^{*}, N, z\right)$ was introduced in (40). In particular, estimate (45) holds.

This concludes the proof of Lemma 3.

\subsubsection{Fixed Point argument}

In this paragraph, we end the proof of the null controllability of system (35), by performing a fixed point argument to the following application: to each $z \in W^{1, \infty}((0, T) \times(0,1))$ such that (40) holds, we associate $y$ solution of (38) given by lemma 3. More precisely, let us first define the set of controls:

$$
A(z)=\left\{\left(\widetilde{v}_{1}, \widetilde{v}_{2}\right) \in W^{1, \infty}(0, T)^{2}: y \text { solution of (38) satisfies }\left.y\right|_{t=T}=0 \text { and } \widetilde{v}_{1}, \widetilde{v}_{2} \text { satisfy }(42)\right\} .
$$

Then, $\Lambda(z)=y$, where $y$ fulfills system (38) for some controls $\left(\widetilde{v}_{1}, \widetilde{v}_{2}\right) \in A(z)$.

Let us recall Kakutani's fixed point theorem (see, for instance, [2]):

Theorem 2. Let $Z$ be a Hilbert space and let $\Lambda: Z \mapsto Z$ be a set-valued mapping satisfying the following assumptions:

1. $\Lambda(z)$ is a nonempty closed convex set of $Z$ for every $z \in Z$.

2. There exists a nonempty convex compact set $E \subset Z$ such that $\Lambda(E) \subset E$.

3. $\Lambda$ is upper-hemicontinuous in $Z$, i.e. for each $\sigma \in Z^{\prime}$ the single-valued mapping

$$
z \mapsto \sup _{y \in \Lambda(z)}\langle\sigma, y\rangle_{Z^{\prime}, Z}
$$

is upper-semicontinuous.

Then $\Lambda$ possesses a fixed point in the set $E$, i.e. there exists $z \in E$ such that $z \in \Lambda(z)$.

Let us check that Kakutani's theorem can be applied to $\Lambda$ and

$$
Z=H^{3 / 4}\left(0, T ; L^{2}(0,1)\right) \cap L^{2}\left(0, T ; H^{7 / 4}(0,1)\right) .
$$

Observe that $Z \subset L^{\infty}(Q) \cap L^{2}\left(0, T ; W^{1, \infty}(0,1)\right)$.

- The fact that $\Lambda(z)$ is a nonempty closed convex set of $Z$ for every $z \in Z$ is very easy to verify, so we leave it to the reader.

- Let us prove that $\Lambda$ maps a compact set into itself. For this, we consider the Hilbert space

$$
H=H^{1}\left(0, T ; L^{2}(0,1)\right) \cap L^{2}\left(0, T ; H^{2}(0,1)\right) .
$$

Then, we introduce the space

$$
E=\left\{w \in H:\|w\|_{H} \leq e^{-K_{0} N /(5 \nu)}\right\},
$$


where $K_{0}$ is the constant in (32). Observe that, in particular, if $w \in E$ then $\|w\|_{\infty} \leq N$ as long as $\nu \in\left(0, \nu_{0}\right)$.

It is very easy to check that $E$ is a compact set of $Z$. Moreover, since $E \subset L^{2}\left(0, T ; W^{1, \infty}(0,1)\right)$, one can prove that for each $z \in E$ the solution $y$ of (38) belongs to $H$ and there exists a constant $C>0$ such that

$$
\|y\|_{H} \leq C\left(\left\|\widetilde{v}_{1}\right\|_{W^{1, \infty}(0, T)}+\left\|\widetilde{v}_{2}\right\|_{W^{1, \infty}(0, T)}+\left\|y^{0}\right\|_{W^{1, p}(0,1)}\right) .
$$

Indeed, let us consider the following lifting of the boundary conditions:

$$
V(t, x)=(1-x) \widetilde{v}_{1}(t)+x \widetilde{v}_{2}(t) \quad t \in(0, T), x \in(0,1) .
$$

By introducing $w:=y-V$, our problem (38) is transformed into

$$
\begin{cases}w_{t}-\nu w_{x x}+((N+z(t, x) / 2) w)_{x}=f(t, x) & \text { in }(0, T) \times(0,1), \\ \left.w\right|_{x=0}=0,\left.\quad w\right|_{x=1}=0 & \text { in }(0, T) \\ \left.w\right|_{t=0}=y^{0}-(1-x) \widetilde{v}_{1}(0)+x \widetilde{v}_{2}(0) & \text { in }(0,1)\end{cases}
$$

where

$$
f(t, x)=(1-x) \widetilde{v}_{1, t}(t)+x \widetilde{v}_{2, t}(t)+(N / 2+z(t, x))\left(-\widetilde{v}_{1}(t)+\widetilde{v}_{2}(t)\right)+z_{x}(t, x) V .
$$

This is a linear parabolic equation with $L_{t}^{2}\left(L_{x}^{\infty}\right)$ coefficient for the zero order term, $L^{\infty}(Q)$ coefficient for the first order (in space) term, $L^{\infty}(Q)$ right hand side and $W^{1, \infty}(0,1)$ initial condition. In this situation, it is not difficult to prove that the solution of (56) belongs to $H$ and (55) holds. (Observe that, thanks to (47), the initial data in (56) satisfies the required compatibility condition.)

Now, looking at the definition of $D$ given in (40), we see that as long as $T>4.3 / N$, we have

$$
D(T, N, z) \geq 0 \quad \forall z \in E, \nu \in\left(0, \nu_{0}\right)
$$

Then, from (55) and taking into account estimates (32) and (42), we obtain for some $C>0$

$$
\|y\|_{H} \leq C\left(e^{-K_{0} / \nu}+e^{-K_{0} /(3 \nu)}\right) \leq e^{-K_{0} N /(5 \nu)} \quad \nu \in\left(0, \nu_{0}\right)
$$

and so $y \in E$.

- It remains to check that $\Lambda$ is upper-hemicontinuous. Thus, assume that $\sigma \in Z^{\prime}$ and let a sequence $\left\{z_{n}\right\}$ be given, with $z_{n} \rightarrow z$ strongly in $Z$. We must prove that

$$
\overline{\lim }_{n \rightarrow+\infty} \sup _{y \in \Lambda\left(z_{n}\right)}\langle\sigma, y\rangle_{Z^{\prime}, Z} \leq \sup _{y \in \Lambda(z)}\langle\sigma, y\rangle_{Z^{\prime}, Z}
$$

Let $\left\{z_{n^{\prime}}\right\}$ be a subsequence of $\left\{z_{n}\right\}$ such that

$$
\varlimsup_{n \rightarrow+\infty} \sup _{y \in \Lambda\left(z_{n}\right)}\langle\sigma, y\rangle_{Z^{\prime}, Z}=\lim _{n^{\prime} \rightarrow+\infty} \sup _{y \in \Lambda\left(z_{n^{\prime}}\right)}\langle\sigma, y\rangle_{Z^{\prime}, Z}
$$

Since each $\Lambda\left(z_{n^{\prime}}\right)$ is a compact set of $Z$, for every $n^{\prime}$ we have

$$
\sup _{y \in \Lambda\left(z_{n^{\prime}}\right)}\langle\sigma, y\rangle_{Z^{\prime}, Z}=\left\langle\sigma, y_{n^{\prime}}\right\rangle_{Z^{\prime}, Z}
$$

for some $y_{n^{\prime}} \in \Lambda\left(z_{n^{\prime}}\right)$. On the other hand, since all the states $y_{n^{\prime}}$ belong to the same compact set $E$, at least for a new subsequence (again indexed by $n^{\prime}$ ), we must have $y_{n^{\prime}} \rightarrow y$ strongly in Z. We will now prove that $y \in \Lambda(z)$. This will achieve the proof of the upper hemicontinuity of $\Lambda$.

Indeed, it can be assumed that the controls $\widetilde{v}_{1, n^{\prime}}$ and $\widetilde{v}_{2, n^{\prime}}$ converge to some functions $\widetilde{v}_{1}$ and $\widetilde{v}_{2}$ weakly-* in $W^{1, \infty}(0, T)$. Then, $y$ solves $(38)$ and $\left.y\right|_{t=T}=0$. Moreover, since inequality (42) 
is independent of $n, \widetilde{v}_{1}$ and $\widetilde{v}_{2}$ also satisfy (42). Therefore, $\left(\widetilde{v}_{1}, \widetilde{v}_{2}\right) \in A(z)$. Consequently, it is immediate that $y$ is the solution to (38) associated to the controls $\widetilde{v}_{1}$ and $\widetilde{v}_{2}$.

This shows that $y \in \Lambda(z)$ and, therefore, $\Lambda$ is upper hemicontinuous.

Consequently, Kakutani's Theorem applies and this implies that there exists $y \in \Lambda(y)$, that is to say, we have found a function $y$ solution of (35) such that (36) and (37) (thanks to (42)) are satisfied. The proof of Proposition 4 is finished.

\section{Proof of Proposition 2}

Again relying on the invariance of the solutions of (1) by the transformation $u(t, x) \leftrightarrow-u(t, 1-x)$, we can always assume that $M>0$. Now Proposition 2 is proven approximately as Proposition 1, but here a (viscous) rarefaction wave is used in place of a traveling wave. More precisely, we start from $N$ which can be chosen larger than $M$. First, we reach $M$ approximately and then we reach $M$ exactly by using the same argument as above.

\subsection{Reaching $M$ approximately}

Let us prove that

Proposition 5. One can find controls $v_{1}$ and $v_{2}$ such that the solution of (1)-(3) with initial condition $u_{\mid t=0}=N$ satisfies for some constant $C>0$ independent from $M, N$ and $\nu$ :

$$
\|u(t, \cdot)-M\|_{W^{1, \infty}(0,1)} \leq C M \sqrt{\nu t} \exp \left\{-\frac{M^{2}}{4 \nu}\left(t-\frac{2}{M}\right)\right\}
$$

for any $t>\frac{2}{M}$ and moreover, the controls satisfy independently from $\nu$ :

$$
\left\|v_{1}\right\|_{L^{\infty}(0, T)}+\left\|v_{2}\right\|_{L^{\infty}(0, T)} \leq N
$$

Proof of Proposition 5. In this situation, the solution $u$ is obtained by taking the restriction to $[0, T] \times[0,1]$ of the solution defined on the whole space domain $\mathbb{R}$ as the unique solution with initial condition:

$$
u(0, x):=\hat{u}_{0}=\left\{\begin{array}{l}
M \text { if } x \leq 0 \\
N \text { if } x>0
\end{array}\right.
$$

Then, $v_{1}$ and $v_{2}$ are obtained by taking the traces of $u$ along the lines $(0, T) \times\{0\}$ and $(0, T) \times\{1\}$. As before, (58) follows directly from the maximum principle so we only have to check (57). form:

In a first step we only consider the $L^{\infty}$ norm. The solution $u(t, x)$ has the following explicit

$$
u(t, x)=\frac{\int_{-\infty}^{0} \frac{x-y}{t} \exp \left\{-\frac{1}{2 \nu}\left(\frac{(x-y)^{2}}{2 t}+M y\right)\right\} d y+\int_{0}^{+\infty} \frac{x-y}{t} \exp \left\{-\frac{1}{2 \nu}\left(\frac{(x-y)^{2}}{2 t}+N y\right)\right\} d y}{\int_{-\infty}^{0} \exp \left\{-\frac{1}{2 \nu}\left(\frac{(x-y)^{2}}{2 t}+M y\right)\right\} d y+\int_{0}^{+\infty} \exp \left\{-\frac{1}{2 \nu}\left(\frac{(x-y)^{2}}{2 t}+N y\right)\right\} d y}
$$

We note that

$$
\begin{aligned}
\int_{-\infty}^{0} \frac{x-y}{t} \exp \left\{-\frac{1}{2 \nu}\left(\frac{(x-y)^{2}}{2 t}\right.\right. & +M y)\} d y \\
& =2 \nu \exp \left(-\frac{x^{2}}{4 \nu t}\right)+M \int_{-\infty}^{0} \exp \left\{-\frac{1}{2 \nu}\left(\frac{(x-y)^{2}}{2 t}+M y\right)\right\} d y
\end{aligned}
$$


(as seen by adding and subtracting $M$ inside the integral). In the same way, we have

$$
\begin{aligned}
& \int_{0}^{+\infty} \frac{x-y}{t} \exp \left\{-\frac{1}{2 \nu}\left(\frac{(x-y)^{2}}{2 t}+N y\right)\right\} d y \\
& \quad=-2 \nu \exp \left(-\frac{x^{2}}{4 \nu t}\right)+N \int_{0}^{+\infty} \exp \left\{-\frac{1}{2 \nu}\left(\frac{(x-y)^{2}}{2 t}+N y\right)\right\} d y
\end{aligned}
$$

Hence we get that

$$
u(t, x)-M=\frac{(N-M) \int_{0}^{+\infty} \exp \left\{-\frac{1}{2 \nu}\left(\frac{(x-y)^{2}}{2 t}+N y\right)\right\} d y}{\int_{-\infty}^{0} \exp \left\{-\frac{1}{2 \nu}\left(\frac{(x-y)^{2}}{2 t}+M y\right)\right\} d y+\int_{0}^{+\infty} \exp \left\{-\frac{1}{2 \nu}\left(\frac{(x-y)^{2}}{2 t}+N y\right)\right\} d y} .
$$

Note that $\hat{u}_{0}$ is non-decreasing, hence $u(t, \cdot)$ is also non-decreasing (as seen from Lemma 1 , and comparing the solutions corresponding to $\hat{u}_{0}$ and $\hat{u}_{0}(\cdot+h)$ ). Using the fact that $\hat{u}_{0} \geq M$, we deduce together with the maximum principle, that $u \geq M$. Consequently, it is sufficient to have an upper estimate for $u(t, 1)-M$. Now from (28) we have

$$
\begin{aligned}
& \int_{-\infty}^{0} \exp \left\{-\frac{1}{2 \nu}\left(\frac{(1-y)^{2}}{2 t}+M y\right)\right\} d y=\sqrt{4 \nu t} \exp \left(\frac{M(M t-2)}{4 \nu}\right) \int_{-\infty}^{\xi_{M}} e^{-s^{2}} d s \\
& \int_{0}^{+\infty} \exp \left\{-\frac{1}{2 \nu}\left(\frac{(1-y)^{2}}{2 t}+N y\right)\right\} d y=\sqrt{4 \nu t} \exp \left(\frac{N(N t-2)}{4 \nu}\right) \int_{\xi_{N}}^{+\infty} e^{-s^{2}} d s
\end{aligned}
$$

with

$$
\xi_{M}:=\frac{M t-1}{2 \sqrt{\nu t}} \text { and } \xi_{N}:=\frac{N t-1}{2 \sqrt{\nu t}} .
$$

We deduce

$$
\begin{aligned}
u(t, 1)-M & \leq \frac{(N-M) \int_{0}^{+\infty} \exp \left\{-\frac{1}{2 \nu}\left(\frac{(1-y)^{2}}{2 t}+N y\right)\right\} d y}{\int_{-\infty}^{0} \exp \left\{-\frac{1}{2 \nu}\left(\frac{(1-y)^{2}}{2 t}+M y\right)\right\} d y} \\
& \leq \frac{(N-M) \exp \left(\frac{M(M t-2)}{4 \nu}\right) \int_{-\infty}^{\xi_{M}} e^{-s^{2}} d s}{\exp \left(\frac{N(N t-2)}{4 \nu}\right) \int_{\xi_{N}}^{+\infty} e^{-s^{2}} d s} \\
& \leq \frac{(N-M) \exp \left(\frac{M(M-2)}{4 \nu}\right) \int_{-\infty}^{\xi_{M}} e^{-s^{2}} d s}{\sqrt{\pi} / 2} .
\end{aligned}
$$

With (30), we get

$$
u(t, 1)-M \leq \frac{2 \sqrt{\nu t}}{\sqrt{\pi}} \frac{N-M}{N t-1} \exp \left\{\frac{-1}{4 \nu t}\right\} \exp \left\{-\frac{M^{2}}{4 \nu}\left(t-\frac{2}{M}\right)\right\},
$$

and the result in $L^{\infty}$ norm follows using $t>2 / M$.

The $L^{\infty}$ estimate on $\partial_{x} u$ is done approximately as in Paragraph 2.1, by using Lemma 2. We fix $x \in[0,1]$; we get

$$
\begin{aligned}
\partial_{x} u(t, x) & =\frac{1}{2 \nu} \frac{\int_{-\infty}^{+\infty} \frac{y-x}{t}\left(\hat{u}_{0}(y)-M\right) \exp \left\{-\frac{1}{2 \nu}\left[\frac{(x-y)^{2}}{2 t}+\int_{0}^{y} \hat{u}_{0}(\eta) d \eta\right]\right\} d y}{\int_{-\infty}^{+\infty} \exp \left\{-\frac{1}{2 \nu}\left[\frac{(x-y)^{2}}{2 t}+\int_{0}^{y} \hat{u}_{0}(\eta) d \eta\right]\right\} d y} \\
& +\frac{1}{2 \nu} \frac{\int_{-\infty}^{+\infty} \frac{y-x}{t}(M-u(t, x)) \exp \left\{-\frac{1}{2 \nu}\left[\frac{(x-y)^{2}}{2 t}+\int_{0}^{y} \hat{u}_{0}(\eta) d \eta\right]\right\} d y}{\int_{-\infty}^{+\infty} \exp \left\{-\frac{1}{2 \nu}\left[\frac{(x-y)^{2}}{2 t}+\int_{0}^{y} \hat{u}_{0}(\eta) d \eta\right]\right\} d y} \\
& =: A+B .
\end{aligned}
$$


Using again the fact that $u(t, \cdot)$ is non-decreasing, we see that we only have to give an upper bound for $\partial_{x} u$. The second term $B$ satisfies

$$
B=\frac{1}{2 \nu}(M-u(t, x)) u(t, x),
$$

and thus is clearly non-positive as follows from the maximum principle. Therefore, it remains to estimate the first term $A$. To this aim, we estimate the denominator from below as follows

$$
\begin{aligned}
\int_{-\infty}^{+\infty} \exp \left\{-\frac{1}{2 \nu}\left[\frac{(x-y)^{2}}{2 t}+\int_{0}^{y} \hat{u}_{0}(\eta) d \eta\right]\right\} d y & \geq \int_{-\infty}^{0} \exp \left\{-\frac{1}{2 \nu}\left[\frac{(x-y)^{2}}{2 t}+\int_{0}^{y} \hat{u}_{0}(\eta) d \eta\right]\right\} d y \\
& =\int_{-\infty}^{0} \exp \left\{-\frac{1}{2 \nu}\left[\frac{(x-y)^{2}}{2 t}+M y\right]\right\} d y \\
& \geq \sqrt{\pi \nu t} \exp \left\{\frac{M}{2 \nu}\left(\frac{M t}{2}-x\right)\right\}
\end{aligned}
$$

For the numerator, thanks to (29), we have

$$
\begin{aligned}
\mathcal{N} & :=\int_{0}^{+\infty} \frac{y-x}{t}\left(\hat{u}_{0}(y)-M\right) \exp \left\{-\frac{1}{2 \nu}\left[\frac{(x-y)^{2}}{2 t}+\int_{0}^{y} \hat{u}_{0}(\eta) d \eta\right]\right\} d y \\
& \leq \int_{0}^{+\infty} \frac{y}{t}(N-M) \exp \left\{-\frac{1}{2 \nu}\left[\frac{(x-y)^{2}}{2 t}+N y\right]\right\} d y \\
& \leq \frac{N-M}{t}\left[2 \sqrt{\nu t}(x-N t) \exp \left\{\frac{N(-2 x+N t)}{4 \nu}\right\} \int_{\frac{-x+N t}{2 \sqrt{\nu t}}}^{+\infty} e^{-s^{2}} d s+2 \nu t \exp \left\{-\frac{x^{2}}{4 \nu t}\right\}\right] \\
& =\frac{N-M}{t} \exp \left\{\frac{N(-2 x+N t)}{4 \nu}\right\}\left[2 \sqrt{\nu t}(x-N t) \int_{\frac{-x+N t}{2 \sqrt{\nu t}}}^{+\infty} e^{-s^{2}} d s+2 \nu t \exp \left\{-\frac{(x-N t)^{2}}{4 \nu t}\right\}\right]
\end{aligned}
$$

Simple integrations by parts prove that

$$
-\int_{y}^{+\infty} e^{-s^{2}} d s+\frac{e^{-y^{2}}}{2 y} \leq \frac{e^{-y^{2}}}{4 y^{3}} .
$$

Plugging (60) with $y=\frac{-x+N t}{2 \sqrt{\nu t}}$ in the previous estimate of $\mathcal{N}$ and using $N t \geq 1 \geq x$, we deduce

$$
\mathcal{N} \leq 4 \frac{N-M}{(N t-1)^{2}} \nu^{2} t \exp \left\{\frac{-x^{2}}{4 \nu t}\right\}
$$

Using $N>M$, we finally obtain

$$
\mathcal{N} \leq 4 M \nu^{2} t
$$

which yields the result.

\subsection{Reaching $M$ exactly}

Reaching $M$ exactly is done exactly as for reaching $N$ exactly (see paragraph 2.2 ). This is due to the fact that we did not use the size of $N$ in paragraph 2.2. This ends the proof of Proposition 2 and hence of Theorem 1.

\section{Technical Results}

\subsection{Proof of the dissipation result}

In this first paragraph, we will prove the estimate presented in (50):

$$
\left\|\left.\varphi\right|_{t=t_{0}}\right\|_{L^{2}(0,1)} \leq \exp \left\{-\frac{\left(\left(N-\|z\|_{\infty} / 2\right) t^{*}-1\right)^{2}}{4 \nu t^{*}} e^{-2\left\|z_{x}\right\|_{L_{t}^{1}\left(L_{x}^{\infty}\right)}}\right\}\left\|\left.\varphi\right|_{t=t_{0}+t^{*}}\right\|_{L^{2}(0,1)}
$$


for any $t_{0} \in\left(0, T^{*}\right)$ and any $t^{*} \in\left(0, T^{*}-t_{0}\right)$ such that (51) is satisfied. Here, $\varphi$ designs the solution of the system

$$
\begin{cases}-\varphi_{t}-\nu \varphi_{x x}-(N+z(t, x) / 2) \varphi_{x}=0 & \text { in }\left(0, T^{*}\right) \times(0,1), \\ \left.\varphi\right|_{x=0}=0,\left.\quad \varphi\right|_{x=1}=0 & \text { in }\left(0, T^{*}\right), \\ \left.\varphi\right|_{t=T^{*}}=\varphi^{0} & \text { in }(0,1) .\end{cases}
$$

Let us first define a function $\Theta(t, x)=e^{\theta(t, x)}$, where $\theta \in L^{\infty}\left(0, T^{*} ; W^{1, \infty}(0,1)\right)$ will be chosen later on. We regard the equation satisfied by $\Theta \varphi$ and after an integration by parts, we obtain

$$
-\frac{1}{2} \frac{d}{d t} \int_{0}^{1}\left|e^{\theta} \varphi\right|^{2} d x+\nu \int_{0}^{1}\left|\left(e^{\theta} \varphi\right)_{x}\right|^{2} d x=\nu \int_{0}^{1}\left|\Theta \theta_{x} \varphi\right|^{2} d x \leq \nu\left\|\theta_{x}\right\|_{\infty}^{2} \int_{0}^{1}\left|e^{\theta} \varphi\right|^{2} d x .
$$

We choose the function $\theta(t, x)=g\left(\psi^{-1}(t, x)\right)$, where $\psi$ is the backward flow associated to $N+$ $z(t, x) / 2$, that is to say,

$$
\left\{\begin{array}{l}
\frac{d \psi}{d t}(t, x)=N+z(t, \psi(t, x)) / 2 \\
\left.\psi\right|_{t=T^{*}}=x
\end{array}\right.
$$

and $g(y)=r_{0} \min \{R,|y|\}$, with $r_{0}, R>0$ two constants which will be chosen later on.

With this choice, after an application of Gronwall lemma in the time interval $\left(t_{0}, t_{0}+t^{*}\right)$, we find the following from (63):

$$
\int_{0}^{1}\left|e^{\theta} \varphi\right|^{2}\left(t_{0}\right) d x \leq \exp \left\{2 \nu r_{0}^{2} t^{*} \exp \left(2\left\|z_{x}\right\|_{L_{t}^{1}\left(L_{x}^{\infty}\right)}\right)\right\} \int_{0}^{1}\left|e^{\theta} \varphi\right|^{2}\left(t_{0}+t^{*}\right) d x .
$$

Here, we have used the expression of $g$ together with the estimate

$$
\left|\psi_{x}^{-1}(t, x)\right|^{2} \leq \exp \left\{2 \int_{0}^{T^{*}}\left\|z_{x}(s)\right\|_{\infty} d s\right\} \quad t \in(0, T), x \in(0,1) .
$$

Now, from the expression of $\psi^{-1}(t, x)$ we observe that

$$
\left|\psi^{-1}\left(t_{0}, x\right)\right| \geq\left(N-\|z\|_{\infty} / 2\right) t_{0} \quad x \in(0,1)
$$

and

$$
\left|\psi^{-1}\left(t_{0}+t^{*}, x\right)\right| \leq 1-\left(N-\|z\|_{\infty} / 2\right)\left(t_{0}+t^{*}\right) \quad x \in(0,1) .
$$

Then, taking a sufficiently large $R>0$ in the definition of $g$, from (64) we find that

$$
\int_{0}^{1}|\varphi|^{2}\left(t_{0}\right) d x \leq C\left(r_{0}, t^{*}\right) \int_{0}^{1}|\varphi|^{2}\left(t_{0}+t^{*}\right) d x
$$

with

$$
C=\exp \left\{2 \nu r_{0}^{2} t^{*} \exp \left(2\left\|z_{x}\right\|_{L_{t}^{1}\left(L_{x}^{\infty}\right)}\right)+2 r_{0}\left(1-\left(N-\|z\|_{\infty} / 2\right) t^{*}\right)\right\} .
$$

Finally, we choose

$$
r_{0}=\frac{e^{-2\left\|z_{x}\right\|_{L_{t}^{1}\left(L_{x}^{\infty}\right)}}\left(\left(N-\|z\|_{\infty} / 2\right) t^{*}-1\right)}{2 \nu t^{*}}
$$

and we find the desired inequality (61) squared. 


\subsection{Proof of the Carleman inequality}

In this last paragraph of the paper, we will provide the proof of the Carleman inequality which was presented in (52). In order to prove this estimate, we follow the steps of the proof in [5].

Hence, let us first perform a change of variables in order to restrict ourselves to the case where $\nu=1$ :

$$
\left\{\begin{array}{l}
\tilde{t}=\nu t \\
\widetilde{x}=x
\end{array}\right.
$$

In the new variables, we have, with $\widetilde{\varphi}(\widetilde{t}, \widetilde{x}):=\varphi(t, x)$ and $\widetilde{z}(\widetilde{t}, \widetilde{x})=z(t, x)$,

$$
\begin{cases}\widetilde{\varphi}_{\tilde{t}}+\widetilde{\varphi}_{\widetilde{x} \widetilde{x}}+\nu^{-1} N \widetilde{\varphi}_{\widetilde{x}}=-\nu^{-1}(\widetilde{z}(\widetilde{t}, \widetilde{x}) / 2) \widetilde{\varphi}_{\widetilde{x}} & (\widetilde{t}, \widetilde{x}) \in\left(0, \nu T^{*}\right) \times(0,1), \\ \widetilde{\varphi}(\widetilde{t}, 0)=\widetilde{\varphi}(\widetilde{t}, 1)=0 & \widetilde{t} \in\left(0, \nu T^{*}\right), \\ \widetilde{\varphi}\left(\nu T^{*}, \widetilde{x}\right)=\widetilde{\varphi}^{0}(\widetilde{x}) & \widetilde{x} \in(0,1) .\end{cases}
$$

Let

$$
\begin{gathered}
\widetilde{N}:=\frac{N}{\nu}, \\
\widetilde{T}:=\nu T^{*} .
\end{gathered}
$$

Then, condition (41) implies

$$
\widetilde{N} \widetilde{T} \geqslant 3 / 2
$$

Let us define a weight function, similar to the one introduced by Fursikov and Imanuvilov in $[8]$

$$
\alpha(\widetilde{t}, \widetilde{x}):=\frac{\beta(\widetilde{x})}{\widetilde{T}-\widetilde{t}}(\widetilde{t}, \widetilde{x}) \in(0, \widetilde{T}) \times(0,1),
$$

where $0 \leqslant \beta \in C^{2}([0,1])$ will be chosen below. We also introduce the function

$$
\psi:=e^{-\alpha} \widetilde{\varphi}
$$

which verifies

$$
P_{1} \psi+P_{2} \psi=P_{3} \psi
$$

with

$$
\begin{gathered}
P_{1} \psi:=\psi_{\widetilde{x} \widetilde{x}}+\alpha_{\widetilde{x}}^{2} \psi+\widetilde{N} \alpha_{\widetilde{x}} \psi+\alpha_{\widetilde{t}} \psi, \\
P_{2} \psi:=\psi_{\widetilde{t}}+2 \alpha_{\widetilde{x}} \psi_{\widetilde{x}}+\widetilde{N} \psi_{\widetilde{x}}, \\
P_{3} \psi:=-\alpha_{\widetilde{x} \widetilde{x}} \psi-\nu^{-1}(\widetilde{z}(\widetilde{t}, \widetilde{x}) / 2)\left(\alpha_{\widetilde{x}} \psi+\psi_{\widetilde{x}}\right) .
\end{gathered}
$$

We develop here the classical proof, consisting in taking the $L^{2}$ norm in identity (72) and then develop all the double products:

$$
\left\|P_{1} \psi\right\|_{L^{2}(Q)}^{2}+\left\|P_{2} \psi\right\|_{L^{2}(Q)}^{2}+2\left(P_{1} \psi, P_{2} \psi\right)_{L^{2}(Q)}=\left\|P_{3} \psi\right\|_{L^{2}(Q)}^{2},
$$

where $Q$ stands for the open set $(0, \widetilde{T}) \times(0,1)$.

Let us compute $2\left(P_{1} \psi, P_{2} \psi\right)_{L^{2}(Q)}$. Let us first compute the terms concerning $\psi_{\widetilde{x} \widetilde{x}}$. We have

$$
\left(\psi_{\widetilde{x} \widetilde{x}}, \psi_{\widetilde{t}}\right)_{L^{2}(Q)}=\frac{1}{2} \int_{0}^{1}\left|\psi_{\widetilde{x}}(0, \widetilde{x})\right|^{2} d \widetilde{x} .
$$

Moreover

$$
\left.2\left(\psi_{\widetilde{x} \widetilde{x}}, \alpha_{\widetilde{x}} \psi_{\widetilde{x}}\right)_{L^{2}(Q)}=\int_{0}^{\widetilde{T}}\left(\alpha_{\widetilde{x}} \widetilde{t}, 1\right)\left|\psi_{\widetilde{x}}(\widetilde{t}, 1)\right|^{2}-\alpha_{\widetilde{x}}(\widetilde{t}, 0)\left|\psi_{\widetilde{x}}(\widetilde{t}, 0)\right|^{2}\right) d \widetilde{t}-\iint_{Q} \alpha_{\widetilde{x} \widetilde{x}}\left|\psi_{\widetilde{x}}\right|^{2} d \widetilde{x} d \widetilde{t} .
$$


Finally,

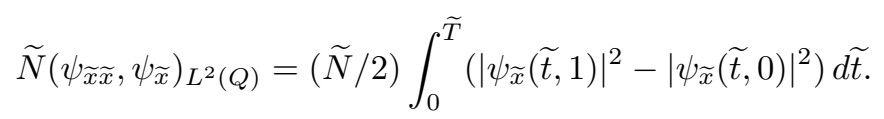

As long as the term $\alpha_{\widetilde{x}}^{2} \psi$ is concerned, we first have

$$
\left(\alpha_{\widetilde{x}}^{2} \psi, \psi_{\widetilde{t}}\right)_{L^{2}(Q)}=-\iint_{Q} \alpha_{\widetilde{x}} \alpha_{\widetilde{x} \tilde{t}}|\psi|^{2} d \widetilde{x} d \widetilde{t}-\frac{1}{2} \int_{0}^{1} \alpha_{\widetilde{x}}^{2}(0, \widetilde{x})|\psi(0, \widetilde{x})|^{2} d \widetilde{x} .
$$

Next,

$$
2\left(\alpha_{\widetilde{x}}^{2} \psi, \alpha_{\widetilde{x}} \psi_{\widetilde{x}}\right)_{L^{2}(Q)}=-3 \iint_{Q} \alpha_{\widetilde{x} \widetilde{x}} \alpha_{\widetilde{x}}^{2}|\psi|^{2} d \widetilde{x} d \widetilde{t}
$$

Finally,

$$
\widetilde{N}\left(\alpha_{\widetilde{x}}^{2} \psi, \psi_{\widetilde{x}}\right)_{L^{2}(Q)}=-\widetilde{N} \iint_{Q} \alpha_{\widetilde{x} \widetilde{x}} \alpha_{\widetilde{x}}|\psi|^{2} d \widetilde{x} d \widetilde{t} .
$$

Let us next perform the terms concerning $\widetilde{N} \alpha_{\widetilde{x}} \psi$. First, we have

$$
\widetilde{N}\left(\alpha_{\widetilde{x}} \psi, \psi_{\widetilde{t}}\right)_{L^{2}(Q)}=-(\widetilde{N} / 2) \iint_{Q} \alpha_{\widetilde{x} \widetilde{t}}|\psi|^{2} d \widetilde{x} d \widetilde{t}-(\widetilde{N} / 2) \int_{0}^{1} \alpha_{\widetilde{x}}(0, \widetilde{x})|\psi(0, \widetilde{x})|^{2} d \widetilde{x} .
$$

Then, we find

$$
2 \widetilde{N}\left(\alpha_{\widetilde{x}} \psi, \alpha_{\widetilde{x}} \psi_{\widetilde{x}}\right)_{L^{2}(Q)}=-2 \widetilde{N} \iint_{Q} \alpha_{\widetilde{x}} \alpha_{\widetilde{x} \widetilde{x}}|\psi|^{2} d \widetilde{x} d \widetilde{t}
$$

The last term provides

$$
\widetilde{N}^{2}\left(\alpha_{\widetilde{x}} \psi, \psi_{\widetilde{x}}\right)_{L^{2}(Q)}=-\left(\widetilde{N}^{2} / 2\right) \iint_{Q} \alpha_{\widetilde{x} \widetilde{x}}|\psi|^{2} d \widetilde{x} d \widetilde{t} .
$$

Lastly, we deal with the computations of the term $\alpha_{\tilde{t}} \psi$. First, we obtain

$$
\left(\alpha_{\widetilde{t}} \psi, \psi_{\widetilde{t}}\right)_{L^{2}(Q)}=-(1 / 2) \iint_{Q} \alpha_{\widetilde{t}}|\psi|^{2} d \widetilde{x} d \widetilde{t}-(1 / 2) \int_{0}^{1} \alpha_{\widetilde{t}}(0, \widetilde{x})|\psi(0, \widetilde{x})|^{2} d \widetilde{x} .
$$

Additionally, we find

$$
\left(\alpha_{\widetilde{t}} \psi, 2 \alpha_{\widetilde{x}} \psi_{\widetilde{x}}\right)_{L^{2}(Q)}=-\iint_{Q}\left(\alpha_{\widetilde{t}} \alpha_{\widetilde{x} \widetilde{x}}+\alpha_{\widetilde{t} \widetilde{x}} \alpha_{\widetilde{x}}\right)|\psi|^{2} d \widetilde{x} d \widetilde{t}
$$

Finally,

$$
\left(\alpha_{\widetilde{t}} \psi, \widetilde{N} \psi_{\widetilde{x}}\right)_{L^{2}(Q)}=-(\widetilde{N} / 2) \iint_{Q} \alpha_{\widetilde{t} \widetilde{x}}|\psi|^{2} d \widetilde{x} d \widetilde{t} .
$$

Putting all these computations together, we conclude that the double product term is

$$
\begin{aligned}
& 2\left(P_{1} \psi, P_{2} \psi\right)_{L^{2}(Q)} \\
& \left.=\int_{0}^{1}\left|\psi_{\widetilde{x}}(0, \widetilde{x})\right|^{2} d \widetilde{x}+\left.\int_{0}^{\widetilde{T}}\left(\left(2 \alpha_{\widetilde{x}}(\widetilde{t}, 1)+\widetilde{N}\right)\left|\psi_{\widetilde{x}}(\widetilde{t}, 1)\right|^{2}-\left(2 \alpha_{\widetilde{x}}(\widetilde{t}, 0)+\widetilde{N}\right) \mid \psi_{\widetilde{x}} \widetilde{t}, 0\right)\right|^{2}\right) d \widetilde{t} \\
& -2 \iint_{Q} \alpha_{\widetilde{x} \widetilde{x}}\left|\psi_{\widetilde{x}}\right|^{2} d \widetilde{x} d \widetilde{t}-4 \iint_{Q} \alpha_{\widetilde{x}} \alpha_{\widetilde{x} \tilde{t}}|\psi|^{2} d \widetilde{x} d \widetilde{t}-\int_{0}^{1} \alpha_{\widetilde{x}}^{2}(0, \widetilde{x})|\psi(0, \widetilde{x})|^{2} d \widetilde{x} \\
& -6 \iint_{Q} \alpha_{\widetilde{x} \widetilde{x}} \alpha_{\widetilde{x}}^{2}|\psi|^{2} d \widetilde{x} d \widetilde{t}-2 \widetilde{N} \iint_{Q} \alpha_{\widetilde{x} \tilde{t}}|\psi|^{2} d \widetilde{x} d \widetilde{t}-\widetilde{N} \int_{0}^{1} \alpha_{\widetilde{x}}(0, \widetilde{x})|\psi(0, \widetilde{x})|^{2} d \widetilde{x} \\
& -6 \widetilde{N} \iint_{Q} \alpha_{\widetilde{x}} \alpha_{\widetilde{x} \widetilde{x}}|\psi|^{2} d \widetilde{x} d \widetilde{t}-\widetilde{N}^{2} \iint_{Q} \alpha_{\widetilde{x} \widetilde{x}}|\psi|^{2} d \widetilde{x} d \widetilde{t} \\
& -\iint_{Q} \alpha_{\widetilde{t} \widetilde{t}}|\psi|^{2} d \widetilde{x} d \widetilde{t}-\int_{0}^{1} \alpha_{\widetilde{t}}(0, \widetilde{x})|\psi(0, \widetilde{x})|^{2} d \widetilde{x}-2 \iint_{Q} \alpha_{\widetilde{t}} \alpha_{\widetilde{x} \widetilde{x}}|\psi|^{2} d \widetilde{x} d \widetilde{t}
\end{aligned}
$$


On the other hand, we have the following for the right hand side term:

$$
\left\|P_{3} \psi\right\|_{L^{2}(Q)}^{2} \leq \iint_{Q}\left(2 \alpha_{\widetilde{x} \widetilde{x}}^{2}|\psi|^{2}+\nu^{-2}|\widetilde{z}(\widetilde{t}, \widetilde{x})|^{2}\left(\alpha_{\widetilde{x}}^{2}|\psi|^{2}+\left|\psi_{\widetilde{x}}\right|^{2}\right)\right) d \widetilde{x} d \widetilde{t}
$$

Combining (74)-(75) with (73), we obtain

$$
\begin{aligned}
& \int_{0}^{\widetilde{T}}\left(2 \alpha_{\widetilde{x}}(\widetilde{t}, 1)+\widetilde{N}\right)\left|\psi_{\widetilde{x}}(\widetilde{t}, 1)\right|^{2} d \widetilde{t}-2 \iint_{Q} \alpha_{\widetilde{x} \widetilde{x}}\left|\psi_{\widetilde{x}}\right|^{2} d \widetilde{x} d \widetilde{t}-6 \iint_{Q} \alpha_{\widetilde{x} \widetilde{x}} \alpha_{\widetilde{x}}^{2}|\psi|^{2} d \widetilde{x} d \widetilde{t} \\
& -2 \iint_{Q} \alpha_{\widetilde{t}} \alpha_{\widetilde{x} \widetilde{x}}|\psi|^{2} d \widetilde{x} d \widetilde{t}-6 \widetilde{N} \iint_{Q} \alpha_{\widetilde{x}} \alpha_{\widetilde{x} \widetilde{x}}|\psi|^{2} d \widetilde{x} d \widetilde{t}-\widetilde{N}^{2} \iint_{Q} \alpha_{\widetilde{x} \widetilde{x}}|\psi|^{2} d \widetilde{x} d \widetilde{t} \\
& \quad \leqslant \iint_{Q}\left(2 \alpha_{\widetilde{x} \widetilde{x}}^{2}|\psi|^{2}+\nu^{-2}|\widetilde{z}(\widetilde{t}, \widetilde{x})|^{2}\left(\alpha_{\widetilde{x}}^{2}|\psi|^{2}+\left|\psi_{\widetilde{x}}\right|^{2}\right)\right) d \widetilde{x} d \widetilde{t}+\int_{0}^{\widetilde{T}}\left(2 \alpha_{\widetilde{x}}(\widetilde{t}, 0)+\widetilde{N}\right)\left|\psi_{\widetilde{x}}(\widetilde{t}, 0)\right|^{2} d \widetilde{t} \\
& \quad+4 \iint_{Q} \alpha_{\widetilde{x}} \alpha_{\widetilde{x} \tilde{t}}|\psi|^{2} d \widetilde{x} d \widetilde{t}+2 \widetilde{N} \iint_{Q} \alpha_{\widetilde{x} \widetilde{t}}|\psi|^{2} d \widetilde{x} d \widetilde{t}+\iint_{Q} \alpha_{\widetilde{t}}|\psi|^{2} d \widetilde{x} d \widetilde{t} \\
& \quad+\int_{0}^{1} \alpha_{\widetilde{x}}^{2}(0, \widetilde{x})|\psi(0, \widetilde{x})|^{2} d \widetilde{x}+\widetilde{N} \int_{0}^{1} \alpha_{\widetilde{x}}(0, \widetilde{x})|\psi(0, \widetilde{x})|^{2} d \widetilde{x}+\int_{0}^{1} \alpha_{\widetilde{t}}(0, \widetilde{x})|\psi(0, \widetilde{x})|^{2} d \widetilde{x}
\end{aligned}
$$

From the definition of $\alpha$ (given in (71)), we find

$$
\begin{aligned}
& \int_{0}^{\widetilde{T}}\left(2 \frac{\beta^{\prime}(1)}{\widetilde{T}-\widetilde{t}}+\widetilde{N}\right)\left|\psi_{\widetilde{x}}(\widetilde{t}, 1)\right|^{2} d \widetilde{t}-2 \iint_{Q} \frac{\beta^{\prime \prime}(\widetilde{x})}{\widetilde{T}-\widetilde{t}}\left|\psi_{\widetilde{x}}\right|^{2} d \widetilde{x} d \widetilde{t}-6 \iint_{Q} \frac{\beta^{\prime \prime}(\widetilde{x})\left(\beta^{\prime}(\widetilde{x})\right)^{2}}{(\widetilde{T}-\widetilde{t})^{3}}|\psi|^{2} d \widetilde{x} d \widetilde{t} \\
& -2 \iint_{Q} \frac{\beta(\widetilde{x}) \beta^{\prime \prime}(\widetilde{x})}{(\widetilde{T}-\widetilde{t})^{3}}|\psi|^{2} d \widetilde{x} d \widetilde{t}-6 \widetilde{N} \iint_{Q} \frac{\beta^{\prime}(\widetilde{x}) \beta^{\prime \prime}(\widetilde{x})}{(\widetilde{T}-\widetilde{t})^{2}}|\psi|^{2} d \widetilde{x} d \widetilde{t}-\widetilde{N}^{2} \iint_{Q} \frac{\beta^{\prime \prime}(\widetilde{x})}{\widetilde{T}-\widetilde{t}}|\psi|^{2} d \widetilde{x} d \widetilde{t} \\
& \quad \leqslant \nu^{-2}\|z\|_{\infty}^{2} \iint_{Q}\left|\psi_{\widetilde{x}}\right|^{2} d \widetilde{x} d \widetilde{t}+\iint_{Q} \frac{2\left(\beta^{\prime \prime}(\widetilde{x})\right)^{2}+\nu^{-2}\|z\|^{2}\left(\beta^{\prime}(\widetilde{x})\right)^{2}}{(\widetilde{T}-\widetilde{t})^{2}}|\psi|^{2} d \widetilde{x} d \widetilde{t} \\
& \quad+\int_{0}^{\widetilde{T}}\left(2 \frac{\beta^{\prime}(0)}{\widetilde{T}-\widetilde{t}}+\widetilde{N}\right)\left|\psi_{\widetilde{x}}(\widetilde{t}, 0)\right|^{2} d \widetilde{t}+4 \iint_{Q} \frac{\left(\beta^{\prime}(\widetilde{x})\right)^{2}}{(\widetilde{T}-\widetilde{t})^{3}}|\psi|^{2} d \widetilde{x} d \widetilde{t}+2 \widetilde{N} \iint_{Q} \frac{\beta^{\prime}(\widetilde{x})}{(\widetilde{T}-\widetilde{t})^{2}}|\psi|^{2} d \widetilde{x} d \widetilde{t} \\
& \quad+2 \iint_{Q} \frac{\beta(\widetilde{x})}{(\widetilde{T}-\widetilde{t})^{3}}|\psi|^{2} d \widetilde{x} d \widetilde{t}+\frac{1}{\widetilde{T}^{2}} \int_{0}^{1}\left(\beta^{\prime}(\widetilde{x})\right)^{2}|\psi(0, \widetilde{x})|^{2} d \widetilde{x}+\frac{\widetilde{N}}{\widetilde{T}} \int_{0}^{1} \beta^{\prime}(\widetilde{x})|\psi(0, \widetilde{x})|^{2} d \widetilde{x} \\
& \quad+\frac{1}{\widetilde{T}^{2}} \int_{0}^{1} \beta(\widetilde{x})|\psi(0, \widetilde{x})|^{2} d \widetilde{x} .
\end{aligned}
$$

Let us now define the function $\beta:[0,1] \mapsto \mathbb{R}$. Let $\delta \in(0,1)$. We take the function satisfying

$$
\beta^{\prime \prime}(\widetilde{x})=-\frac{1}{1-\delta} \frac{2\left(\beta^{\prime}(\widetilde{x})\right)^{2}+\beta(\widetilde{x})}{3\left(\beta^{\prime}(\widetilde{x})\right)^{2}+\beta(\widetilde{x})} \quad \widetilde{x} \in[0,1],
$$

together with the initial conditions $\beta(0)=\delta$ and $\beta^{\prime}(0)=0.807$. One can check, for instance with Matlab, that, if $\delta>0$ is small enough,

$$
\begin{gathered}
\beta>0, \beta^{\prime}>0 \text { and } \beta^{\prime \prime}<0 \text { on }[0,1], \\
\beta(1)<0.435 .
\end{gathered}
$$


We choose a $\delta>0$ such that(78) and (79) hold. From (76), (77) and (78), we have

$$
\begin{aligned}
& -2 \iint_{Q} \frac{\beta^{\prime \prime}(\widetilde{x})}{\widetilde{T}-\widetilde{t}}\left|\psi_{\widetilde{x}}\right|^{2} d \widetilde{x} d \widetilde{t}-6 \delta \iint_{Q} \beta^{\prime \prime}(\widetilde{x}) \frac{\beta(\widetilde{x})+3\left(\beta^{\prime}(\widetilde{x})\right)^{2}}{(\widetilde{T}-\widetilde{t})^{3}}|\psi|^{2} d \widetilde{x} d \widetilde{t} \\
& -6 \widetilde{N} \iint_{Q} \frac{\beta^{\prime}(\widetilde{x}) \beta^{\prime \prime}(\widetilde{x})}{(\widetilde{T}-\widetilde{t})^{2}}|\psi|^{2} d \widetilde{x} d \widetilde{t}-\widetilde{N}^{2} \iint_{Q} \frac{\beta^{\prime \prime}(\widetilde{x})}{\widetilde{T}-\widetilde{t}}|\psi|^{2} d \widetilde{x} d \widetilde{t} \\
& \quad \leqslant \nu^{-2}\|z\|_{\infty}^{2} \iint_{Q}\left|\psi_{\widetilde{x}}\right|^{2} d \widetilde{x} d \widetilde{t}+\iint_{Q} \frac{2\left(\beta^{\prime \prime}(\widetilde{x})\right)^{2}+\nu^{-2}\|z\|^{2}\left(\beta^{\prime}(\widetilde{x})\right)^{2}}{(\widetilde{T}-\widetilde{t})^{2}}|\psi|^{2} d \widetilde{x} d \widetilde{t} \\
& \quad+\int_{0}^{\widetilde{T}}\left(2 \frac{\beta^{\prime}(0)}{\widetilde{T}-\widetilde{t}}+\widetilde{N}\right)|\psi \widetilde{x}(\widetilde{t}, 0)|^{2} d \widetilde{t}+2 \widetilde{N} \iint_{Q} \frac{\beta^{\prime}(\widetilde{x})}{(\widetilde{T}-\widetilde{t})^{2}}|\psi|^{2} d \widetilde{x} d \widetilde{t} \\
& \quad+\frac{1}{\widetilde{T}^{2}} \int_{0}^{1}\left(\beta^{\prime}(\widetilde{x})\right)^{2}|\psi(0, \widetilde{x})|^{2} d \widetilde{x}+\frac{\widetilde{N}}{\widetilde{T}} \int_{0}^{1} \beta^{\prime}(\widetilde{x})|\psi(0, \widetilde{x})|^{2} d \widetilde{x}+\frac{1}{\widetilde{T}^{2}} \int_{0}^{1} \beta(\widetilde{x})|\psi(0, \widetilde{x})|^{2} d \widetilde{x}
\end{aligned}
$$

Additionally, using (39), the definition of $\widetilde{T}=\nu T^{*}$ and the fact that $\beta^{\prime \prime}(\widetilde{x}) \leqslant-2 /((1-\delta) 3)$, we can absorb the first term in the right hand side of (80) with

$$
-2 \iint_{Q} \frac{\beta^{\prime \prime}(\widetilde{x})}{\widetilde{T}-\widetilde{t}}\left|\psi_{\widetilde{x}}\right|^{2} d \widetilde{x} d \widetilde{t}
$$

as long as $\nu$ is small enough. Furthermore,

$$
\begin{aligned}
-6 \delta \iint_{Q} \beta^{\prime \prime}(\widetilde{x}) & \frac{\beta(\widetilde{x})+3\left(\beta^{\prime}(\widetilde{x})\right)^{2}}{(\widetilde{T}-\widetilde{t})^{3}}|\psi|^{2} d \widetilde{x} d \widetilde{t} \\
& \geqslant \iint_{Q} \frac{2\left(\beta^{\prime \prime}(\widetilde{x})\right)^{2}+\nu^{-2}\|z\|^{2}\left(\beta^{\prime}(\widetilde{x})\right)^{2}}{(\widetilde{T}-\widetilde{t})^{2}}|\psi|^{2} d \widetilde{x} d \widetilde{t}+2 \widetilde{N} \iint_{Q} \frac{\beta^{\prime}(\widetilde{x})}{(\widetilde{T}-\widetilde{t})^{2}}|\psi|^{2} d \widetilde{x} d \widetilde{t}
\end{aligned}
$$

for $\nu \in\left(0, \nu_{0}\right)$.

From (78) and (80), we have

$$
\begin{aligned}
-\widetilde{N}^{2} \iint_{Q} \frac{\beta^{\prime \prime}(\widetilde{x})}{\widetilde{T}-\widetilde{t}}|\psi|^{2} d \widetilde{x} d \widetilde{t} \leqslant \int_{0}^{\widetilde{T}}\left(2 \frac{\beta^{\prime}(0)}{\widetilde{T}-\widetilde{t}}+\widetilde{N}\right)|\psi \widetilde{x}(\widetilde{t}, 0)|^{2} d \widetilde{t}+\frac{1}{\widetilde{T}^{2}} \int_{0}^{1}\left(\beta^{\prime}(\widetilde{x})\right)^{2}|\psi(0, \widetilde{x})|^{2} d \widetilde{x} \\
+\frac{\widetilde{N}}{\widetilde{T}} \int_{0}^{1} \beta^{\prime}(\widetilde{x})|\psi(0, \widetilde{x})|^{2} d \widetilde{x}+\frac{1}{\widetilde{T}^{2}} \int_{0}^{1} \beta(\widetilde{x})|\psi(0, \widetilde{x})|^{2} d \widetilde{x} .
\end{aligned}
$$

Let us recall that $\psi:=e^{-\alpha} \widetilde{\varphi}$. Then, from (70), (71), (78) and (81), we deduce that

$$
\widetilde{N} \iint_{Q} \frac{1}{\widetilde{T}-\widetilde{t}} e^{-2 \alpha}|\widetilde{\varphi}|^{2} d \widetilde{x} d \widetilde{t} \leqslant C\left(\int_{0}^{\widetilde{T}}|\widetilde{\varphi} \widetilde{x}(\widetilde{t}, 0)|^{2} d \widetilde{t}+\frac{1}{\widetilde{T}} \int_{0}^{1}|\widetilde{\varphi}(0, \widetilde{x})|^{2} d \widetilde{x}\right) .
$$

In (82) and in the sequel, $C$ will stand for generic positive constants independent of $\nu, N, T^{*}$ and $\varphi^{0}$.

By (71) and (78), $e^{-2 \alpha}$ reaches its minimum in the region $[2 \widetilde{T} / 3,(2+3 \gamma) \widetilde{T} / 3] \times[0,1]$ at $(\widetilde{t}, \widetilde{x})=(2 \widetilde{T} / 3,1)($ recall that $0<\gamma<1 / 3$ was introduced right before $(52))$. Hence

$$
\frac{\widetilde{N}}{\widetilde{T}} e^{-2 \alpha(2 \widetilde{T} / 3,1)} \int_{0}^{1} \int_{2 \widetilde{T} / 3}^{(2+3 \gamma) \widetilde{T} / 3}|\widetilde{\varphi}|^{2} d \widetilde{t} d \widetilde{x} \leqslant C\left(\int_{0}^{\widetilde{T}}|\widetilde{\varphi} \widetilde{x}(\widetilde{t}, 0)|^{2} d \widetilde{t}+\frac{1}{\widetilde{T}} \int_{0}^{1}|\widetilde{\varphi}(0, \widetilde{x})|^{2} d \widetilde{x}\right) .
$$

From (71), we deduce, with (79),

$$
\exp \{-2 \alpha(2 \widetilde{T} / 3,1)\}=\exp \{-6 \beta(1) / \widetilde{T}\}>\exp \{-(2.61) / \widetilde{T}\} .
$$

Coming back to our original variables (see (66), (68) and (69)), we get from (83) and (84) the desired inequality (52). 


\section{References}

[1] F. Ancona, A. Marson, On the attainable set for scalar nonlinear conservation laws with boundary control. SIAM J. Control Optim. 36 (1998), no. 1, pp. 290-312.

[2] J.-P. Aubin, L'analyse non linéaire et ses motivations économiques, Masson, Paris 1984.

[3] J.-M. Coron, On the controllability of the 2-D incompressible Navier-Stokes equations with the Navier slip boundary conditions. ESAIM Contrôle Optim. Calc. Var. 1 (1995/96), 35-75.

[4] J.-M. Coron, Some open problems on the control of nonlinear partial differential equations, in "Perspectives in Nonlinear Partial Differential Equations: In honour of Haïm Brezis", H. Berestycki, M. Bertsch, B. Peletier, L. Véron eds., Birkaüser, to appear.

[5] J.-M. Coron, S. Guerrero, Singular optimal control: A linear 1-D parabolic-hyperbolic example, Asymp. Anal. 44 3,4 (2005), 237-257.

[6] C.M. Dafermos, Hyperbolic conservation laws in continuum physics, Grundlehren Math. Wissenschaften Series, Vol. 325, Springer Verlag, 2000.

[7] R. Danchin, Poches de tourbillon visqueuses, J. Math. Pures Appl. 76 (1997), 609-647.

[8] A. Fursikov, O. Yu. Imanuvilov, Controllability of Evolution Equations, Lecture Notes \#34, Seoul National University, Korea, 1996.

[9] S. Guerrero, O. Yu. Imanuvilov, Remarks on global controllability for the Burgers equation with two control forces, to appear in Ann. Inst. H. Poincaré, Analyse non lin.

[10] S. Guerrero, G. Lebeau, Singular optimal control for a transport-diffusion equation, preprint 2005.

[11] E. Hopf, The partial differential equation $u_{t}+u u_{x}=\mu u_{x x}$, Comm. Pure Appl. Math. 3 (1950), 201-230.

[12] T. Horsin, On the controllability of the Burgers equation. ESAIM: Control Opt. Calc. Var. 3 (1998), pp. 83-95.

[13] S. N. KRUŽKOv, First order quasilinear equations with several independent variables. (Russian) Mat. Sb. (N.S.) 81 (123) 1970, pp. 228-255. Translated in Math. USSR Sbornik Vol. 10 (1970), No. 2, pp. 217-243. 